\title{
Hétéroptères Miridae de Guyane française : Liste préliminaire, descriptions de taxa nouveaux et données additionnelles
}

\author{
Luis A. A. Costa ${ }^{(1)}$, Frédéric Chérot ${ }^{(2), *} \&$ Diego Carpintero ${ }^{(3)}$ \\ ${ }^{(1)}$ Museu Nacional, Quinta da Boa Vista, Rio de Janeiro 20940-040, Rio de Janeiro, Brazil \\ (2) Université Libre de Bruxelles, Laboratoire de Systématique et d'Ecologie animales, cp 160/13, Avenue F.D. Roosevelt 50, B-1050 Bruxelles, Belgique \\ (3) División de Entomología, Facultad de Ciencias Naturales y Museo, Universidad Nacional de La Plata, Paseo del Bosque s/n, La Plata 1900, \\ Buenos Aires, Argentina» par «División Entomología, Museo Argentino de Ciencias Naturales «Bernardino Rivadavia», \\ Av. Angel Gallardo 470, C1405DJR, Buenos Aires, Argentina \\ * Auteur pour la correspondance
}

\begin{abstract}
The Heteroptera Miridae of the French Guyana: preliminary list, descriptions of new taxa and additionnal data. Two new genera and six new species are described from French Guyana: Diplozona emboliata n. sp., Guianocoris unicolor n. gen, n. sp., Lundioides couturieri n. gen, n. sp. Neella guiana n. sp., Phytocoris guianus n. sp. and Phytocoris jugatus n. sp. The new combination Guianocoris ruber (Carvalho \& Carpintero 1991a) n. comb. is proposed. Sthenaridea carvalhoi Schuh \& Schwartz 1988 and Proboscidotylus nigrosquamis (Maldonado 1969) are redescribed and illustrated. The female genital structures of Iridopeplus pellucidipennis Bergroth 1910 and the male of Henicocnemis luctuosa (Stål 1860) are described for the first time. The variability of Monalonion annulipes Signoret 1858 is analyzed. Fourteen species are quoted as new for French Guyana: Fulvius bisbistillatus (Stål 1860), F. satipoensis Carvalho \& Costa 1994. Henicocnemis luctuosa (Stål 1860), Horcisoides punctatus (Carvalho 1976), Maxacalinus cuiabanus Carvalho 1976, Notholopus coreoides Carvalho 1975, N. cuiabanus Carvalho 1975, Proboscidotylus nigrosquamis (Maldonado 1969), Sthenaridea carvalhoi Schuh \& Schwartz 1988, Taedia bananalensis Carvalho \& Costa 1994, T. cajabiana Carvalho 1976, T. coimbrai Carvalho 1975, T. compactoides Carvalho 1975 and T. xinguana Carvalho 1975. A provisional list of 46 Miridae is provided for the country.
\end{abstract}

Résumé. Deux nouveaux genres et six espèces nouvelles sont décrits de Guyane française, à savoir : Diplozona emboliata n. sp., Guianocoris unicolor n. g., n. sp., Lundioides couturieri n. gen, n. sp. Neella guiana n. sp., Phytocoris guianus n. sp. et Phytocoris jugatus $\mathbf{n}$. sp. La nouvelle combinaison Guianocoris ruber (Carvalho \& Carpintero 1991a) n. comb. est proposée. Deux espèces sont redécrites et illustrées: Sthenaridea carvalhoi Schuh \& Schwartz 1988 et Proboscidotylus nigrosquamis (Maldonado 1969). Les génitalias femelles d'Iridopeplus pellucidipennis Bergroth 1910 sont décrits pour la première fois, ainsi que le mâle d'Henicocnemis luctuosa (Stål 1860). La variabilité de Monalonion annulipes Signoret 1858 est mise en évidence. Quatorze espèces sont signalées comme nouvelles pour la faune de Guyane : Fulvius bisbistillatus (Stål 1860), F. satipoensis Carvalho \& Costa 1994 Henicocnemis luctuosa (Stål 1860), Horcisoides punctatus (Carvalho 1976), Maxacalinus cuiabanus Carvalho 1976, Notholopus coreoides Carvalho 1975, N. cuiabanus Carvalho 1975, Proboscidotylus nigrosquamis (Maldonado 1969). Sthenaridea carvalhoi Schuh \& Schwartz 1988, Taedia bananalensis Carvalho \& Costa 1994, T. cajabiana Carvalho 1976, T. coimbrai Carvalho 1975, T. compactoides Carvalho 1975 et T. xinguana Carvalho 1975, ce qui porte la liste provisoire des Miridae du Département à 46 espèces.

Keywords: Bugs, new genera, new species, South-America.

$\mathrm{L}$ a faune des Hétéroptères Miridae de Guyane française reste très mal connue. Schuh (1995), dans son catalogue des Miridae du monde, ne cite que quatre espèces de Mirinae et aucun Cylapinae de ce territoire. Carvalho et al. (2001), pour leur part, ne mentionnent guère plus d'une vingtaine d'espèces de Miridae de Guyane dont neuf nouvelles pour la science. Aucun de ces chiffres ne peut être considéré

E-mail: tlcosta@uol.com.br, fcherot@ulb.ac.be,dcarpint@macn.gov.ar Accepté le 29 mai 2008 comme représentatif de la richesse spécifique locale, certainement beaucoup plus élevée.

Peu avant son décès en 1994, le $\mathrm{Dr}$ J. M. C. Carvalho avait commencé la rédaction d'un manuscrit concernant certains des taxa inclus dans le présent travail, et ce avec l'aide du premier auteur (L.A.A.Costa). Malheureusement, emporté par une crise d'asthme le 21 octobre 1994, le grand hémiptériste brésilien ne devait jamais achever ce manuscrit. Ayant repris l'étude du matériel en question et disposant de nombreux spécimens de ces espèces et d'autres non encore connues de Guyane française, les coauteurs 
jugèrent intéressant d'en profiter pour dresser une liste préliminaire des Miridae de ce département.

Elle porte à 46 le nombre de Miridae connus de Guyane française, dont : (1) deux genres et six espèces nouveaux pour la science décrits ci-après, à savoir : Diplozona emboliata n. sp., Guianocoris unicolor $\mathbf{n}$. gen, n. sp., Lundioides couturieri n. gen, n. sp., Neella guiana n. sp., Phytocoris guianus n. sp. et Phytocoris jugatus n. sp.; (2) quatorze espèces citées pour la première fois du département, à savoir: Fulvius bisbistillatus (Stål 1860), F. satipoensis Carvalho \& Costa 1994, Henicocnemis luctuosa (Stål 1860), Horcisoides punctatus (Carvalho 1976), Maxacalinus cuiabanus Carvalho 1976, Notholopus coreoides Carvalho 1975, N. cuiabanus Carvalho 1975, Proboscidotylus nigrosquamis (Maldonado 1969), Sthenaridea carvalhoi Schuh \& Schwartz 1988, Taedia bananalensis Carvalho \& Costa 1994, T. cajabiana Carvalho 1976, T. coimbrai Carvalho 1975, T. compactoides Carvalho 1975 et T. xinguana Carvalho 1975.

\section{Matériel et méthode}

Nous admettons une conception nominaliste des entités taxinomiques (Chérot 2002).

Les abréviations suivantes sont utilisées pour désigner diverses institutions ou collections privées : BMNH : Natural History Museum (anciennement British Museum of Natural History), Londres, Grande Bretagne; CAR: collection D. Carpintero (Buenos Aires) ; CFC: collection F. Chérot (Bruxelles); IRSNB : Institut Royal des Sciences naturelles de Belgique, Bruxelles, Belgique ; MLP : Facultad de Cienciads Naturales y Museo de La Plata, Universidad Nacional de La Plata, La Plata, Argentine; MNRJ: Museu Nacional, Rio de Janeiro, Brazil ; USNM : United States National Museum of Natural History, Washington DC, U.S.A.

La terminologie concernant les structures génitales suit partiellement Slater (1950), Davis (1955), Kelton (1955) et Stonedahl (1988), tels que synthétisés par Chérot (2002).

Les mensurations sont exprimées en $\mathrm{mm}$. L'unité n'est pas systématiquement répétée.

Les taxa sont simplement repris dans la liste par ordre alphabétique de leurs nomina, au sein des sous-familles et tribus elles-mêmes classées par ordre alphabétique.

Pour chaque espèce les indications suivantes sont fournies :

(1) Une chrésosynonymie partielle (sensu Dubois 2000) reprenant, outre la référence de la description originale du taxon concerné, les autres références pertinentes pour l'étude de la faune de Guyane française (citation de l'espèce dans le Département, éventuellement compléments à la description originale,...). Une chrésosynonymie complète des Miridae du Monde, réactualisant le catalogue de Schuh (1995) est disponible à l'adresse http://research.amnh.org/pbi/catalog.

(2) La liste des spécimens examinés. Les auteurs ne disposent de coordonnées géographiques précises des lieux de collecte que pour un petit nombre de spécimens parmi ceux examinés, les étiquettes n'en faisant généralement pas mention. Il s'avère délicat - même à l'aide des outils de l'IGN disponibles sur l'Internet
- de déterminer a posteriori ces coordonnées précisément faute d'avoir soi-même procédé aux collectes. Nous donnons ci-après de telles coordonnées, entre crochets, déterminées à l'aide du Géoportail de l'Institut Géographique National et ce pour les holotypes. Une liste des lieux de collecte mentionnés dans l'article, avec leurs coordonnées et leur code sur la carte (fig. 52) et en annexe 2. En dépit de la précision des valeurs fournies, une certaine imprécision demeure néanmoins quant à leur exactitude (notamment en ce qui concerne la Montagne des Singes) et ces coordonnées doivent être interprétées en conséquence, tout comme la carte les représentant.

(3) S'il y a lieu, une description ou une redescription du taxon.

(4) S’il y a lieu, une brève discussion (ou un commentaire sur la distribution).

En outre, une carte globale des localités de collecte mentionnées dans le texte est fournie.

\section{Taxonomie et faunistique}

\section{Bryocorinae}

\section{Dicyphini}

\section{Monalonion annulipes Signoret 1858 (Figs 1-4)}

Références. Monalonion annulipes Signoret 1858 : 500 (comme n. sp.) ; Monalonion reuteri Bergroth 1910 : 67 (comme n. sp., décrite de Guyane française ; synonymie par Carvalho 1959 : 138) ; Monalonion annulipes: Schuh 1995 : 517-518 (catalogue).

Spécimens examinés. Brésil : 10ิ, 1q : Rondônia, IV.1998, Olzeno T. leg. (CFC). Guyane française : 19 : Route Départementale numéro 6 vers Kaw, 33,5 km S.E. de Roura, 15.II.1986, G. Tavakilian (étiqueté par Carvalho comme holotype de Monalonion femoralis n. sp., un nom manuscrit)

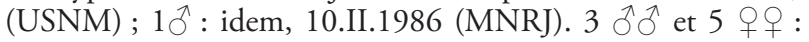
Route Nationale numéro 2 vers Regina, $67 \mathrm{~km}$ de Cayenne, 4.VI.1986, E. G. Riley \& D. A. Rider. 2 우 : Route Nationale numéro 2 vers Regina, $45 \mathrm{~km} \mathrm{~S}$. de Cayenne, 31.V.1986, E. G. Riley \& D. A. Rider. 10 : extension de la route numéro 1,20 km S.E. de Saint Laurent, 07.VI.1986, E. G. Riley \& D. A. Rider. 1 ㅇ : Kaw, 20.VII.1985, G. Tavakilian. 1 đ : Montagne des Singes, près de Kourou, 3.VI.1986, E. G. Riley \& D. A. Rider (tous étiquetés par Carvalho comme paratypes de Monalonion femoralis n. sp., un nom manuscrit) (MNRJ). 1 우 : Piste Crique serpent, point kilométrique 19,1, (piège) malaise, VI.2001, J. Cerda leg. (FC nº 4172) (MNHN).

Commentaire. Monalonion annulipes Signoret 1858 est une espèce bien connue, tant d'Amérique Centrale (du Mexique, d'où elle fut décrite par Signoret, jusquau Panama en passant par le Costa Rica et le Guatemala) que d'Amérique du Sud (Brésil, Colombie, Equateur, Guyane française, Surinam, Pérou) (Carvalho 1972; Schuh 1995). Carvalho, dans sa révision du genre (1972: 123-127), donne une redescription assez détaillée de ce taxon ainsi que sa synonymie. Il analyse en outre le matériel-type des 
nombreux synonymes plus récents de $M$. annulipes, notamment celui de $M$. reuteri Bergroth 1910 décrit de Guyane française. Son travail permet de se rendre compte de la variabilité de cette espèce, en terme de coloration dorsale (plus particulièrement hémélytrale, mais aussi pronotale, scutellaire, dans une moindre mesure antennaire...), un fait déjà relevé par Distant (1883) et souligné par Carvalho (1972). L'étude des spécimens cités ci-dessus nous a conduits à constater que la forme des métafémurs, en particulier l'importance de l'étranglement médian de ceux-ci, et la densité de leur pilosité, ainsi que la longueur de la pilosité du deuxième article antennaire sont des états de caractères eux aussi assez variables. Les spécimens de Guyane ont fréquemment un étranglement médian des métafémurs plus marqué, une pilosité métafémorale plus dense et une pilosité du deuxième article antennaire moins longue que certains spécimens d'autres provenances. En revanche, leurs structures génitales (figs 2-4) sont totalement identiques à celles connues de $M$. annulipes (se référer à Carvalho 1972, p. 126, figs 6-8). Il ne semble donc pas justifié de considérer les spécimens guyanais comme constituant un taxon séparé («femoralis» sensu Carvalho, nom manuscrit).

\section{Eccritotarsini}

\section{Cyrtocapsus femoralis Reuter 1892}

Références. Cyrtocapsus femoralis Reuter 1892 : 394 (comme n. sp.); Miccus elutipes Bergroth 1910 : 65-67 (comme gen. sp. n. décrits de Guyane française ; synonymie par Carvalho 1954c : 12) ; Cyrtocapsus femoralis: Schuh 1995 : 542 (catalogue).

Remarque. Pas de donnée additionnelle disponible. Décrit du Venezuela (Reuter op. cit.).

\section{Neella guiana n. sp.}

(Figs 5-9)

Matériel type. Guyane française : Holotype $\delta$ : «Hwy (= Highway) ext. N1, $20 \mathrm{~km} \mathrm{SE}$ of St. Laurent, 07.VI.1986, E.

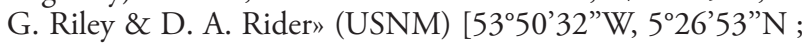
fig. 52, point 7]. Paratypes : 1 ㅇ : «Hwy (= Highway) D6 to Kaw, 34km SE of Roura, point 05-06.VI.1986, E. G. Riley \& D. A. Rider» (MNRJ) ; 10, 1인 «Hwy (= Highway) N2 to Regina, $45 \mathrm{~km} \mathrm{S.} \mathrm{of} \mathrm{Cayenne,} \mathrm{31.V.1986,} \mathrm{E.} \mathrm{G.} \mathrm{Riley} \mathrm{\&} \mathrm{D.} \mathrm{A.}$

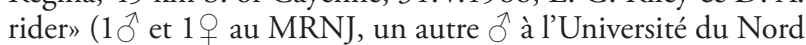
Dakota, United States of America).

Description. Mâle (holotype). Mensurations. Longueur totale : 3,8 ; largeur totale : 1,5 . Longueur de la tête en vue dorsale : 0,3 ; largeur du diatone : 1,0 ; largeur du vertex : 0,5 . Longueur des articles antennaires : I : 0,$3 ;$ II $: 0,8 ;$ III $: 0,5$; IV : 0,6. Longueur du pronotum : 0,5; largeur (postérieure) $\mathrm{du}$ pronotum : 1,2. Longueur du cunéus : 0,7 ; largeur du cunéus : 0,4 . Coloration générale jaune claire à jaune orangé en collection, article II des antennes noir à l'apex, les III et
IV entièrement brun-noirâtre, yeux tachés de noir. Corps finement et superficiellement ponctué (fig. 5), sa pilosité présente mais peu visible, sétiforme, couchée (sauf sur la tête et sur l'anneau apical où elle est semi-dressée), blanche. Tête large. Clypéus invisible en vue dorsale, couvert par le front dont il est nettement séparé, de couleur jaune orangé uniforme. Joues et brides allongées, ni carénées, ni tuberculées, jaune-orangé, nettement pileuses. Front mal délimité du vertex, jauneorangé, non strié, très finement ponctué, pileux, la pilosité semi-dressée, sétiforme, blanche. Vertex très finement ponctué lui aussi, immarginé, dépourvu de sillon ou de concavité, de couleur jaune-orangé comme le front avec une bande orange médiane peu contrastée qui se prolonge sur la partie postérieure du front. Yeux pédonculés, grands, atteignant pratiquement les $3 / 4$ de la hauteur de la tête en vue latérale (hors gula) et occupant un peu moins des $2 / 3$ de la hauteur de la tête en vue frontale,

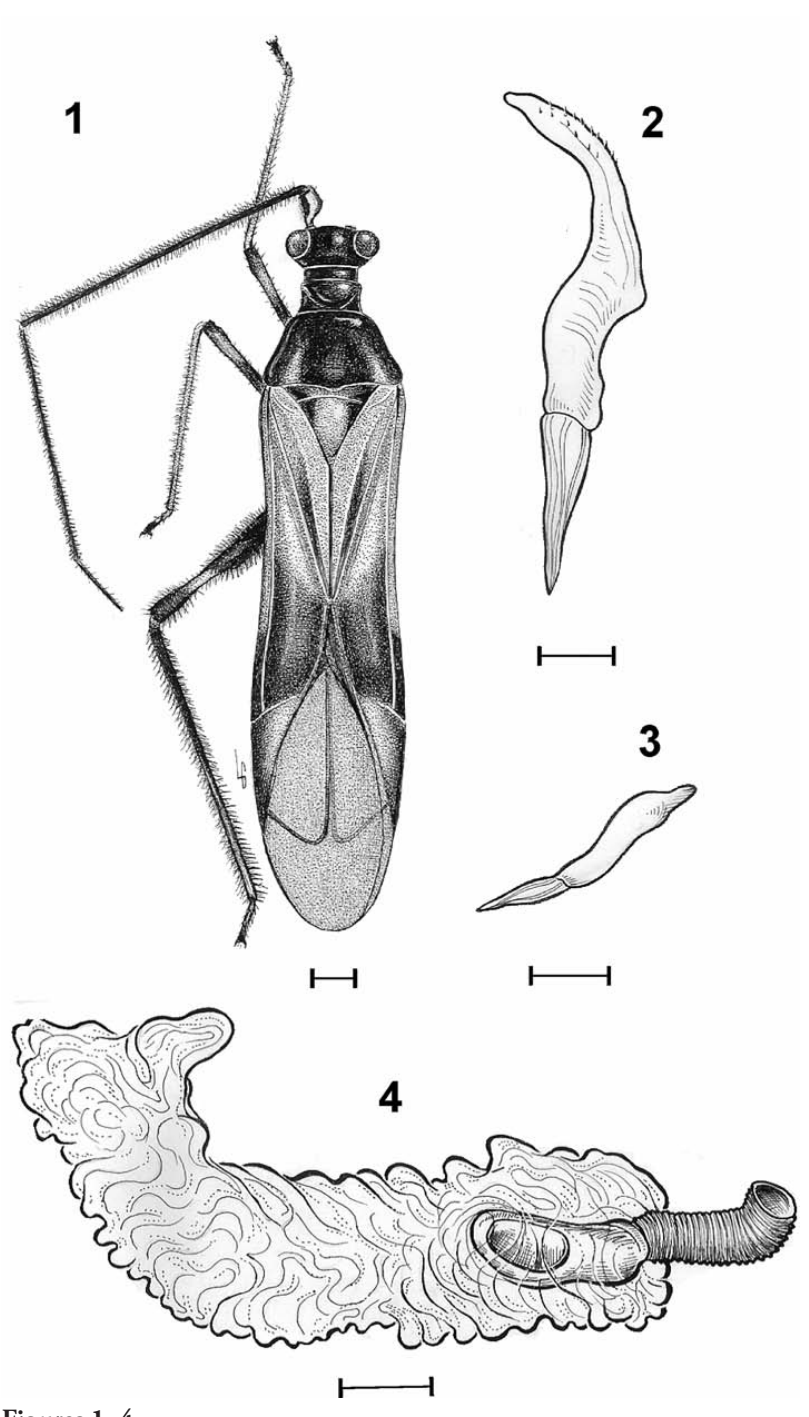

Figures 1-4

Monalonion annulipes Signoret 1858. 1, Habitus en vue dorsale. 2, Paramère gauche en vue dorsale. 3, Paramère droit en vue dorsale. 4, Endophallus en vue latéro-dorsale. [Echelles $=1 \mathrm{~mm}$ (habitus); 0,1 mm (génitalias)]. 
en contact avec le bord antérieur du pronotum, faiblement pileux, de couleur orange brunâtre largement taché de gris noir. Articles I des antennes jaune, porteur de quelques longues soies noires, épineuses, dressées et d'autres soies blanches, sétiformes, semi-dressées. Article II jaune clair dans sa moitié basale, noir apicalement, globalement porteur de soies blanches, sétiformes, semi-dressées. Articles III et IV brun-noirâtre, porteurs du même type de pilosité que l'article II. Rostre jaune clair, atteignant les coxae moyens. Pronotum jaune-orangé uniforme. Anneau apical finement ponctué, la ponctuation superficielle et moyennement large et nettement pileux, la pilosité sétiforme, blanche, semi-dressée. Callosités pratiquement invisibles, seulement marquées par la faible concavité qui les sépare, n'atteignant pas les bords latéraux du pronotum. Disque pronotal portant des soies sétiformes semi-dressées, sa surface nettement ponctuée, les points superficiels mais relativement larges. Bord
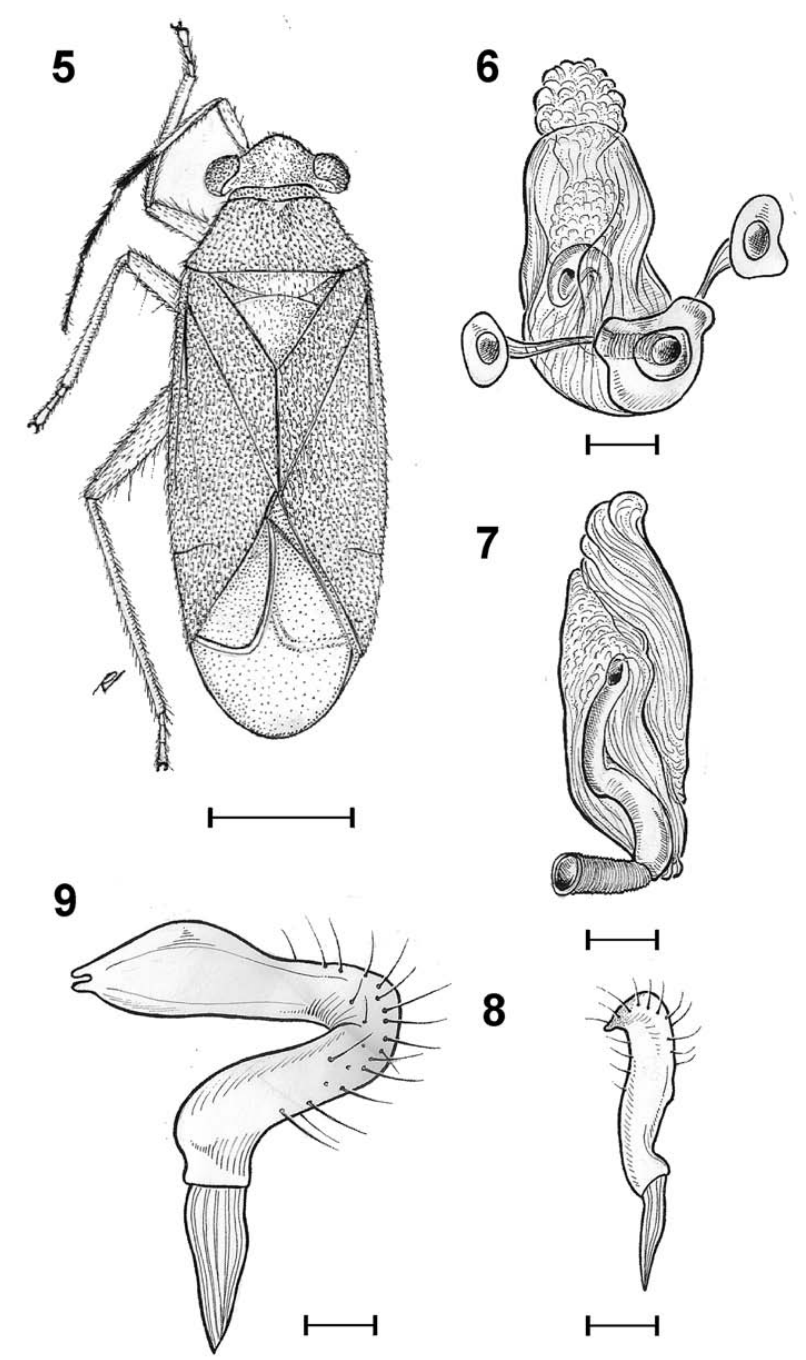

Figures 5-9

Neella guiana n. sp. 5, Habitus en vue dorsale. 6, Phallus en vue dorsolatérale. 7, Endophallus en vue dorso-latérale. 8, Paramère droit en vue ventrale. 9, Paramère gauche en vue dorsale. [Echelles $=1 \mathrm{~mm}$ (habitus); $0,05 \mathrm{~mm}$ (génitalias)]. postérieur du pronotum immarginé, quasi-droit médianement et latéralement. Angles huméraux arrondis. Bords latéraux du pronotum immarginés et sans carène. Scutellum jaune orangé, sa ponctuation et sa pilosité extrêmement réduites. Parascutum largement découvert, jaune orangé, pourvu de fossettes latérales peu marquées. Pattes avec fémurs, tibias et tarses jauneorangé clair, couverts de longues soies sétiformes blanches. Métatibias porteurs en outre d'épines jaunâtres peu distinctes des soies. Hémélytres apparaissant globalement jaune-orangé, clavii jaune-orangé, finement ponctués, endocoria largement translucides incolores, portant une ponctuation relativement large et superficielle, jaune orangée, plus dense et plus ruguleuse vers la suture clavo-coriale, exocoria jaune-orangé uniforme, très finement ponctués. Cunei largement incolores et translucides, jaune-orangé sur leur rebord latéro-externe. Membrane grisbeige, nervures jaunes. Pilosité hémélytrale sétiforme, blanche, semi-dressée, plus dense sur les bords de l'exocorie à laquelle s'ajoutent quelques soies noires, quasi spiniformes, sur les marges latéro-externes des cunei. Face ventrale jaune orangé tachée de brun rouge sur l'abdomen.

Génitalias. Phallus (figs 6-7) simple, le ductus seminis allongé, le gonopore secondaire ovale allongé. Paramère gauche (fig. 9) falciforme, épais, portant de longues soies et une apophyse allongée, large, bifide. Paramère droit (fig. 8) petit.

Femelles (paratypes). Similaires aux mâles en taille et coloration, les yeux bruns.

Derivatio nominis. L'épithète réfère au département de Guyane française dans lequel la nouvelle espèce fut collectée.

Commentaire. Parmi les Neella jaune-orangé unicolores, $N$. guiana n. sp. se distingue de Neella anduzeei Carvalho $1954 \mathrm{~b}$, de $N$. rondonia Carvalho 1985c et de $N$. pallescens Carvalho \& Schaffner 1985 par le deuxième article antennaire jaune basalement et noir apicalement (alors qu'il est complètement noir chez les 3 autres espèces). La nouvelle espèce se distingue en outre de $N$. pallescens par la structure du ductus seminis, beaucoup plus long chez ce dernier taxon (et encore inconnue tant chez $N$. anduzeei que chez $N$. rondonia).

\section{Sysinas fulvicollis (Fabricius 1803)}

Références. Capsus fulvicollis Fabricius 1803 : 244 (comme n. sp.) ; Sysinas fulvicollis: Bergroth 1910: 65 (nov. comb., l'auteur indique que le type provient de Guyane française) ; Schuh 1995: 579 (catalogue).

Remarque. Pas de donnée additionnelle disponible. Connu du Surinam (Carvalho \& Rosas 1965 : 210).

\section{Cylapinae}

\section{Fulviini}

\section{Fulvius amapensis Carvalho \& Costa 1994}

Références. Fulvius amapensis Carvalho \& Costa 1994 : 70-72 (comme n. sp. décrite du Brésil et de Guyane française) ; Carvalho et al. 2001 : 148; Carpintero et al. 2006: 6 (citation d'Argentine); Gorczyca $2006: 42$ (catalogue). 
Remarque. Pas de donnée additionnelle disponible (mais voir la discussion concernant Fulvius satipoensis).

\section{Fulvius bisbistillatus (Stål 1860)}

Références. Cyllecoris bis-bistillatus Stål 1860 : 54 (comme n. sp.) ; Fulvius bisbistillatus: Carvalho \& Costa 1994: 78, 80-82 (redescription, mention du Guyana); Schuh 1995: 26-27 (catalogue); Gorczyca 2006: 43-44 (catalogue, première mention de l'espèce pour la Guyane française); Sadowska-Woda et al. 2006 : 623, 625 (génitalias femelles).

Spécimens examinés. Nicaragua : Zelaya : 1 : : Cerro Salsaya,

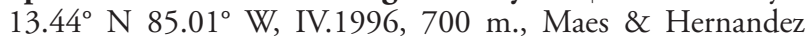
(Muséo entomologico de Léon, Nicaragua). Guyane française : $1 q$ : Patawa, point kilométrique 37, 12.IX.1998, A. Matocq leg. $1 \delta^{\Uparrow}$ : idem précédent, 13.IX.1998 (FC n 4653). 19 : Acournay, Auberge du Bois Diable, 15.IX.1998, A. Matocq leg. $1 \widehat{\jmath}$ : Barrage de Petit Saut, Carbet Maman Lézard, point kilométrique 21, 14.IX.1998, A. Matocq leg. (MNHN).

Commentaire. Espèce largement répandue en Amérique Centrale et en Amérique du Sud, connue d'Argentine, du Brésil, du Chili, de Colombie, de l'Equateur, du Guatemala, du Guyana, du Honduras, du Mexique, du Panama, du Paraguay, du Pérou, de Porto Rico, de République Dominicaine, du Surinam, de Tobago et du Venezuela (Carvalho \& Costa 1994 : 80 ; Gorczyca 2006 : 44). Elle n’a été mentionnée pour la première fois de Guyane française que récemment (Gorczyca 2006 : 43-44). On notera que la base des coxae I à III est plus ou moins nettement tachée selon les spécimens, les coxae ne sont donc pas jaunes unicolores comme la clé de Carvalho \& Costa (op. cit., couplets 15 et 21) pourrait le laisser croire. En outre, l'article II des antennes est quasi complètement jaune sur l'un de nos spécimens. Les génitalias concordent cependant parfaitement avec les descriptions de la littérature.

\section{Fulvius breddini Reuter 1902}

Références. Fulvius breddini Reuter 1902 : 156 (comme n. sp.) ; Carvalho \& Costa 1994 : 82-84 (mention de Guyane française); Schuh 1995 : 27 (catalogue); Gorczyca $2006: 44$ (catalogue); Sadowska-Woda et al. 2006 : 624-625 (génitalias femelles).

Spécimens examinés. Guyane française : $2 \widehat{\jmath}, 2 q 0+$ : Kaw, point kilométrique 37,5, piège malaise, 30.VII au 14.IX.2000, J. Cerda (FC n 4650-4651 et sans nº). 290 : Régina, piste de Bélizon, point kilométrique de la piste (PK) 15, GPS : $4^{\circ} 15^{\prime} 0,7^{\prime \prime} \mathrm{N}, 52^{\circ} 20^{\prime} 88^{\prime \prime} \mathrm{W}$ [sic! recte $52^{\circ} 22^{\prime} 59^{\prime \prime W} 4^{\circ} 17^{\prime} 03^{\prime \prime} \mathrm{E}$ ], 31.I.2003, pièges lumineux, R. Garrouste leg. (MNHN). Nota: Les étiquettes des deux derniers spécimens femelles portent la curieuse mention "p.k. 9,5 de la piste p.k. 15». Selon notre collègue A. Matocq (in litt.), la piste de Bélizon, qui part de la RN2 entre Cayenne et Régina, est carrossable sur une quinzaine de kilomètres et l'endroit mentionné correspondrait bien à un point kilométrique situé à $15 \mathrm{~km}$ de la RN2.

Remarque. Espèce largement répandue en Amérique Centrale et en Amérique du Sud, citée du Brésil, de
Colombie, du Costa Rica, d'Equateur (Galapagos), du Guyana, du Mexique, du Panama, du Paraguay, du Pérou et du Venezuela par Carvalho \& Costa (1994: $84)$ et Gorczyca (2006 : 44).

\section{Fulvius satipoensis Carvalho \& Costa 1994}

Références. Fulvius satipoensis Carvalho \& Costa 1994: 118, 120, 124 (comme n. sp.) ; Gorczyca 2006 : 48-49 (catalogue); Chérot et al. 2007 : 176-177 (génitalias femelles).

Spécimen examiné. Guyane française : $1 q$ : Régina, piste de Bélizon, point kilométrique de la piste PK 15 GPS :

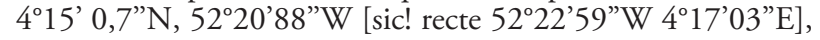
31.I.2003, pièges lumineux, R. Garrouste leg. (MNHN). Nota : même mention que deux Fulvius breddini femelles.

Commentaire. Espèce nouvelle pour la Guyane française, décrite du Pérou et récemment citée du Nicaragua (Chérot et al. 2007: 176). Par ses mensurations et par ses génitalias, ce spécimen semble bien se rattacher au taxon que Chérot et al. (op. cit.) identifiaient comme Fulvius satipoensis et dont ils figuraient le vagin. Cependant, tant que les génitalias femelles de Fulvius amapensis Carvalho \& Costa 1994 n'auront pas été étudiés, un doute subsistera sur l'identification de ce seul spécimen guyanais, les deux espèces se séparant essentiellement par des caractères morphométriques et la structure du paramère gauche. Chérot et al. (op. cit. : 176, figs 1-3, s) signalaient la présence d'un sclérite dorsal surmontant les anneaux pariéto-vaginaux. Il apparaît à l'étude du spécimen de Guyane que ce sclérite est un sclérite unique de la paroi dorsale, couvrant effectivement les anneaux en vue dorsale mais étant indépendant de ceux-ci.

\section{Peritropoides annulatus Carvalho 1955}

Références. Peritropoides annulatus Carvalho 1955: 625 (comme n. sp.) ; Schuh 1995: 34-35 (catalogue); Carvalho et al., 2001 : 157-158 (description du mâle d'après des spécimens de Guyane française); Gorczyca 2006 : 64 (catalogue).

Remarque. Pas de donnée additionnelle disponible.

\section{Peritropoides guianus Carvalho, Costa \& Chérot 2001}

Références. Peritropoides guianus Carvalho, Costa \& Chérot 2001 : 158159 (comme n. sp. décrite de Guyane française) ; Gorczyca 2006: 64 (catalogue).

Remarque. Pas de donnée additionnelle disponible.

\section{Deraeocorinae}

\section{Deraeocorini}

\section{Diplozona emboliata n. sp. (Figs 10-13)}

Matériel type. Guyane française: Holotype $\delta:$ French Guyana: Hwy (= Highway) N2 (= Nationale 2) to Regina, 
67 km of Cayenne, 6.VI.1986, E. G. Riley \& D. A. Rider» (USNM) [52 $19^{\prime} 37^{\prime \prime} \mathrm{W}, 4^{\circ} 25^{\prime} 48^{\prime \prime} \mathrm{N}$; fig. 52, point 25]. Paratypes : 1 र et 1 q : «French Guyana : Hwy (Highway) N2 (Nationale 2) to Regina, $67 \mathrm{~km}$ of Cayenne, 1.VI.1986, E. G. Riley \& D. A. Rider» (MNRJ).

Description. Mâle (holotype). Mensurations. Longueur totale : 3,6 ; largeur totale : 1,6. Longueur de la tête en vue dorsale : 0,2 ; largeur du diatone : 0,8 ; largeur du vertex : 0,2. Longueur des articles antennaires : I : 0,4 ; II : 1,0 ; III : 0,5 ;

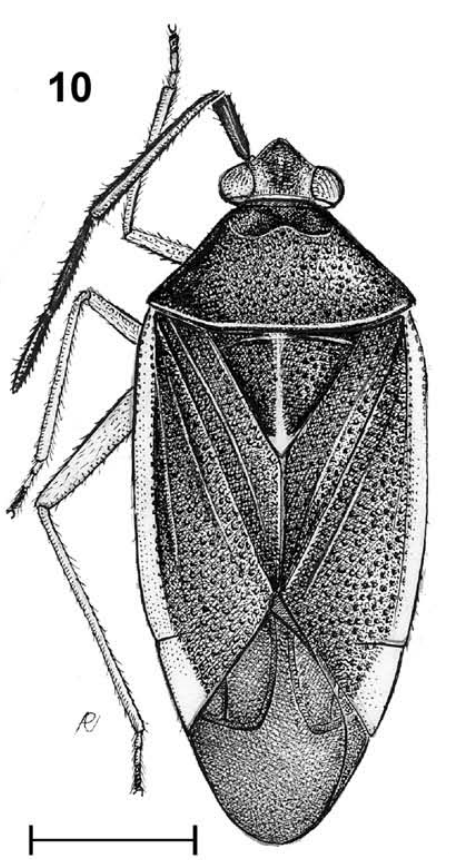

\section{1}
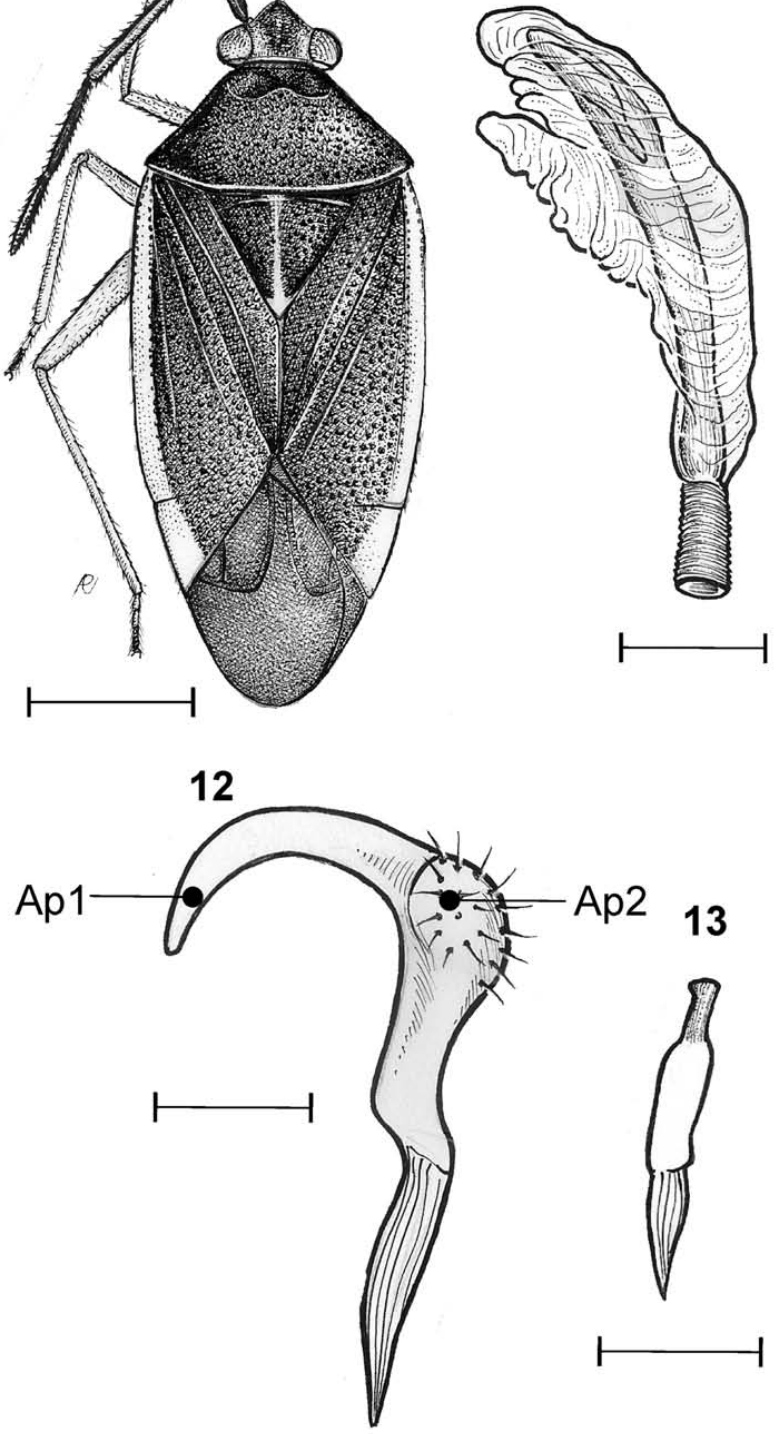

Figures 10-13

Diplozona emboliata n. sp. 10, Habitus en vue dorsale. 11, Endophallus en vue dorso-latérale. 12, Paramère gauche en vue dorsale. Ap1 : apophyse primaire, Ap2 : apophyse secondaire. 13, Paramère droit. [Echelles = $1 \mathrm{~mm}$ (habitus) ; 0,1 $\mathrm{mm}$ (génitalias)].
IV : 0,4. Longueur du pronotum : 0,6; largeur (postérieure) du pronotum : 1,4. Longueur du cunéus : 0,5 ; largeur du cunéus : 0,4. Coloration générale brun foncé, le pronotum uniformément brun, finement bordé de jaune aux angles huméraux mais dépourvu de tâche ou de bande jaune (fig. 10), l'embolium et une large partie latéro-externe du cunéus flaves, translucides. Corps nettement ponctué, sa pilosité présente mais peu visible. Tête. Clypéus libre en vue dorsale, non couvert par une prolongation frontale, de couleur jaune et portant deux lignes brunes sub-latérales allant en se rétrécissant ventralement. Joues et brides ni carénées, ni tuberculées, jauneorangé, les joues bordées dorsalement par une fine ligne noire. Front non strié, très finement ponctué, bien délimité du vertex, avec une tache médiane plus foncée. Vertex très finement ponctué lui aussi, finement marginé, portant médianement une petite concavité peu profonde ainsi qu'une large tache sub-triangulaire brune, sa pointe dirigée vers le pronotum et latéralement une paire de taches triangulaires jaune-orangé sur la marge postéro-interne de chaque œil. Pilosité céphalique restreinte à quelques courtes soies sétiformes couchées. Yeux grands, atteignant pratiquement la marge ventrale de la tête en vue latérale et occupant les $2 / 3$ de la hauteur de la tête en vue frontale, en contact avec le bord antérieur du pronotum, faiblement pileux, de couleur orange-rosé tachés de noir. Articles I, III et IV des antennes brun foncé ou noirs, le II jaune clair, tous les articles épais, en forme de massue, porteur de soies sétiformes semi-dressées. Rostre clair, son apex noir, dépassant les coxae postérieurs. Pronotum brun-noir, à l'exception de ses marges postérieure et latérales, finement lignées de jaune. Anneau apical pratiquement glabre, ni strié, ni ponctué, brun noir, mat, porteur de deux petites taches guttiformes jaunâtres submédianes. Callosités bien marquées, ni striées ni ponctuées, elles aussi brun-noir, pratiquement jointives médianement, nettement séparées des bords latéraux du pronotum. Disque pronotal brun-noir, fortement ponctué, les points noirs, portant des soies sétiformes couchées. Bord postérieur du pronotum finement marginé, quasi-droit médianement, en $S$ allongé latéralement. Angles huméraux arrondis. Bords latéraux du pronotum marginés mais sans carène. Scutellum noir, sauf une ligne médiane et une tache triangulaire, calleuse, à l'apex qui sont jaunâtres; sa ponctuation et sa pilosité identiques à celles du pronotum. Parascutum invisible. Pattes. Fémurs et tibias jaune-orangé clairs, le troisième article des tarses sombre. Métatibias porteurs de spinules noirs, de longues soies sétiformes semi-dressées, et d'épines jaunâtres. Hémélytres noirs, exceptés l'embolium et la moitié externe des cunei blanchâtres, leurs ponctuation et pilosité identiques à celles du pronotum. Membrane brun foncé, nervures noires. Face ventrale noire. Génitalias. Endophallus (fig. 11) restreint, le ductus seminis allongé, le gonopore secondaire ovale allongé. Paramère gauche (fig. 12) falciforme, portant une apophyse secondaire (Ap2) couverte de soies épaisses et une apophyse primaire (Ap1) allongée. Paramère droit (fig. 13) petit.

Femelle (paratype). Similaire au mâle mais légèrement plus grande (longueur totale : 4,4).

Derivatio nominis. L'épithète réfere à un des état de caractères permettant aisément de reconnaître la nouvelle espèce : son embolium jaune claire translucide.

Commentaire. Nos spécimens correspondent parfaitement à la diagnose générique donnée par Carvalho \& Costa (1990 : 176). En résumé, il s’agit de Deraeocorinae relativement larges, peu pileux 
(Carvalho \& Costa, op. cit., disent même glabres), fortement ponctués, au clypéus proéminent, arrondi à l'apex, ayant de grands yeux et des antennes épaisses, dont les différents articles sont en forme de massue et de largeur comparable entre-eux, ainsi qu'un pronotum dont les rebords sont marginés latéralement. Diplozona emboliata n. sp. se distingue des autres espèces du genre à la fois par son pronotum unicolore brun-noir (il est systématiquement porteur de taches chez les autres espèces, voir Carvalho \& Costa, op. cit., p. 184, fig. 32), son embolium jaune clair translucide et par la structure des génitalias du mâle.

\section{Surinamellini}

\section{Eustictus sp. 1}

Spécimen examiné. Guyane française : 1 우 : Piste de Kaw, Patawa, point kilométrique 37, 06.X.2002, piège lumineux, R. Garrouste leg. (MNHN).

\section{Eustictus sp. 2}

Spécimen examiné. Guyane française : 19 : Régina, piste de Bélizon, point kilométrique 9, 5 de la piste PK 15 GPS : $4^{\circ} 15^{\prime} 0,7^{\prime \prime} \mathrm{N}, 52^{\circ} 20^{\prime} 88^{\prime \prime} \mathrm{W}$ [sic! recte $52^{\circ} 22^{\prime} 59^{\prime \prime} \mathrm{W} 4^{\circ} 17^{\prime} 03^{\prime \prime} \mathrm{E}$ ], 31.I.2003, pièges lumineux, R. Garrouste leg. (MNHN). Nota : même remarque que pour Fulvius breddini et $F$ satipoensis.

Remarque. Ainsi que l'écrivaient Chérot et al. (2007: 182) le genre Eustictus devrait être revu avant toute nouvelle description, nombre d'espèces (notamment parmi celles décrites des U.S.A. au début du $20^{\mathrm{ème}}$ siècle) restant mal connues. En attendant, toute identification spécifique s'avère délicate.

\section{Mirinae}

\section{Mirini}

\section{Dagbertus insignis Carvalho 1977}

Références. Dagbertus insignis Carvalho 1977 : 18-19 (comme n. sp.) ; Carvalho 1984a: 101-102 (génitalias mâles); Schuh 1995: 751 (catalogue).

Spécimens examinés. Brésil : Rio: Paratype (?) : 19 : Pétropolis, 1958, Carvalho (MNRJ). Guyane française : $2 \widehat{\delta} \widehat{\delta}$, $1 q$ : Kaw, Patawa, point kilométrique 37,5, 29.IX.2000, A. Matocq leg. (FC n4201-4203). 1 : : Itani, Antécumepata, Saut Kialo, 4-7.XII.1975, M. Boulard, P. Jauffret \& P. Pompanon leg. (FC n ${ }^{\circ}$ 4614) $(\mathrm{MNHN})$. Surinam : $2 \hat{\jmath} \widehat{\jmath}$ : Itani, Carbet Lavaud, rive surinamienne, 03-04.XII.1975, M. Boulard, P. Jauffret \& P. Pompanon leg. (FC ns 4615-4616) (MNHN).

Discussion. Espèce nouvelle pour la Guyane française. Ce superbe petit Mirini fut décrit du Brésil, Mato Grosso (Holotype femelle, Sinop, Rio Teles Pires) et Nova Teutonia (paratypes femelles, pas de localité citée) ainsi que d'Argentine, Entre Rios (paratype femelle de Leibig). L'appartenance du spécimen du MNRJ cité cidessus à la série type est douteuse dans la mesure où il n'est pas mentionné par Carvalho (1977: 19).

\section{Henicocnemis luctuosa (Stål 1860) (Figs 14-18)}

Références. Deraeocoris luctuosus Stål 1860 : 50 (comme n. sp.) ; Calocoris atterimus Poppius 1915: 38-39 (synonymie par Carvalho \& Fontes 1972 : 165); Henicocnemis luctuosus: Schuh 1995 : 775 (catalogue).

Spécimens examinés. Guyane française : $10 \hat{0}$ : Highway Nationale 2 vers Regina, $67 \mathrm{~km}$ de Cayenne, 6.VI.1986, E. G. Riley \& D. A. Rider (USNM, étiqueté comme holotype $\mathrm{d}^{\prime}$ "Henicocnemis tibialis»). Argentine : Misiones : $10^{\Uparrow}$ : Villa Maria, Dept. Concepción, Viana leg. ; 10ิ : A. del Valle (= Aristobulo del Valle), 02.II.1951, Duret leg. (CAR).

Description du sexe mâle. Mensurations (spécimen de Guyane française). Longueur totale : 4,0; largeur totale : 1,8. Longueur de la tête en vue dorsale : 0,2 ; largeur du diatone : 0,8 ; largeur du vertex : 0,3 . Longueur des articles antennaires : I : 0,6 ; II : 1,0 ; III : 0,6 ; IV : 0,6 . Longueur du pronotum : 0,8 ; largeur (postérieure) du pronotum : 1,7. Longueur du cunéus : 0,9; largeur du cunéus : 0,6. Coloration dorsale foncée, orange à rouge-brun (fig. 14). Vertex avec deux petites taches jauneorangé au bord des yeux. Tête allongée en vue frontale. Clypéus libre en vue dorsale, à peine séparé du front par une petite concavité, orange-brunâtre avec une ligne transversale noire à la base d'où partent trois lignes longitudinales noires, une médiane et deux latérales, deux anneaux transversaux noirs, un sub-basal et un médian, et le tylus noir. Brides et joues ni carénées, ni tuberculées, unicolores, orange-brunâtre à brun-rouge. Gula portant de longues soies raides, sétiformes, érigées. Front oblique, lisse, brillant, brun-rouge, portant une petite pilosité sétiforme blanche - argentée sous lumière incidente - couchée ou semi-dressée. Yeux grands, argentés, tachés latéralement et postérieurement d'orange-brun, en contact avec l'anneau apical du pronotum, occupant en vue frontale les $2 / 3$ de la hauteur de la tête et en vue latérale pratiquement toute cette hauteur. Premier article antennaire plus court que la longueur de la tête, en forme de massue, unicolore, rouge-brun foncé, portant quelques courtes soies sétiformes, blanches (apparaissant dorées sous lumière incidente), dressées ou semi-dressées, de longueur voisine de la largeur de l'article à son apex et de nombreuses soies noires, couchées, bien plus courtes encore. Deuxième article antennaire subcylindrique, légèrement épaissi à l'apex, noir avec un anneau jaune à la base, portant des soies sétiformes dressées ou semi-dressées de longueur légèrement supérieure à la largeur de l'article. Troisième article antennaire largement noir, avec un anneau jaune juste après sa base et un autre à son apex. Quatrième article noir. Vertex lisse, brillant, brunrouge foncé, dépourvu de sillon, de concavité ou de marge, avec une petite tache allongée jaune-orangée rebordant la partie postérieure de la marge latéro-interne des yeux. Rostre jauneorangé, atteignant les coxae postérieurs. Pas de cou. Pronotum. Anneau apical noir, brillant, très finement strié transversalement, quasi glabre. Callosités pronotales peu marquées, pratiquement planes, glabres et lisses, oblongues, bien séparées des rebords du pronotum. Disque pronotal brun-rouge bordé de noir, très peu contrasté, brillant, pourvu d'une paire de soies raides à ses angles antérieurs et de soies sétiformes semi-dressées principalement 
sur ses marges latérales, faiblement ponctué, la ponctuation relativement large mais superficielle. Bord postérieur du disque quasi droit médianement, incurvé convexe latéralement, les angles huméraux arrondis à légèrement anguleux. Pas de carène sur les bords latéraux. Scutellum plan, lisse, unicolore brunrouge, portant quelques courtes soies sétiformes couchées ou semi-dressées. Mésoscutum couvert. Pattes. Fémurs jaune pâle, leur pilosité sétiforme, jaune-blanchâtre, semi-dressée. Tibias noirs, les médians et postérieurs porteurs de trois anneaux jaune

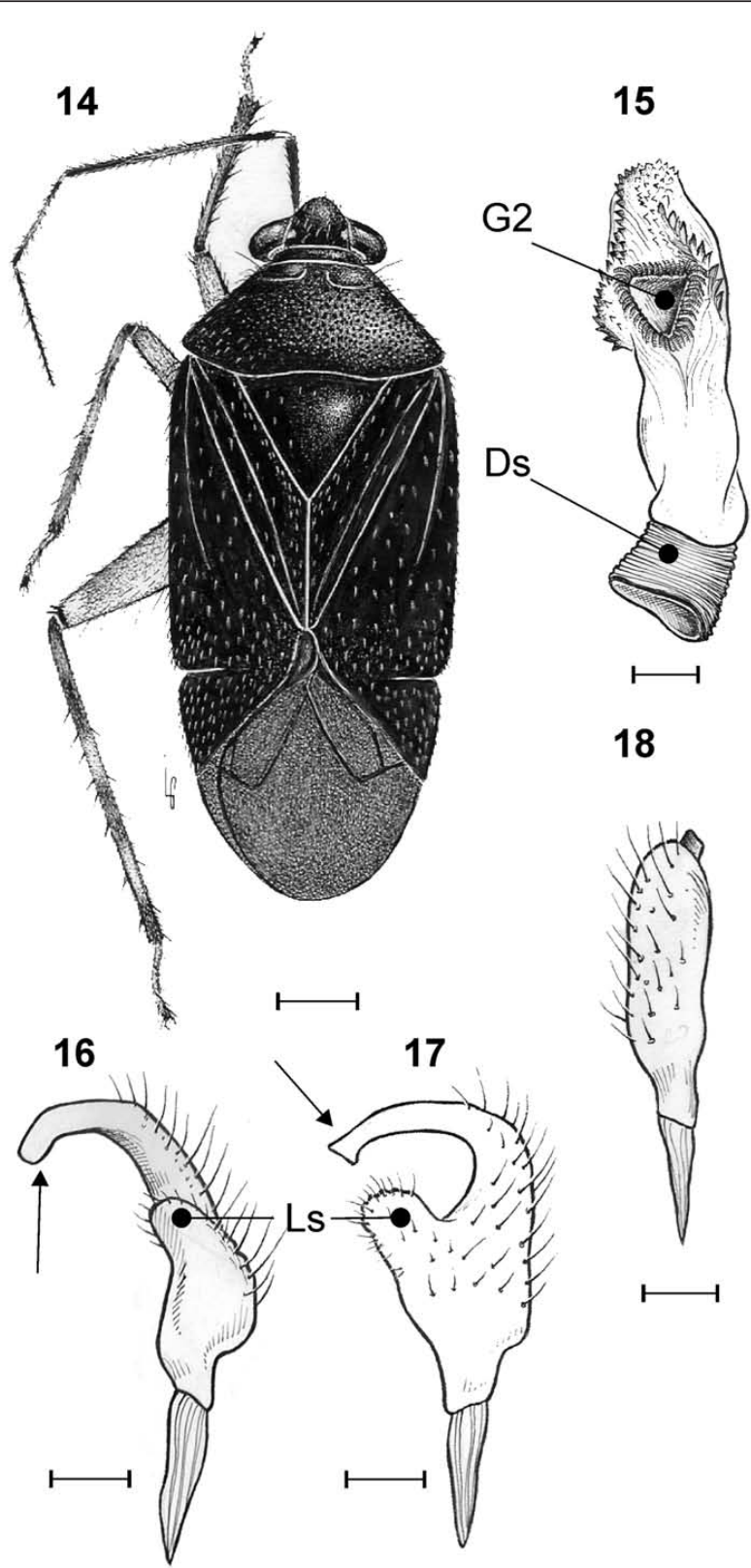

Figures 14-18

Henicocnemis luctuosa (Stål 1860). 14, Habitus en vue dorsale. 15, Endophallus en vue dorso-latérale. 16-17, Paramère gauche sous différentes orientations, flèches : apophyse primaire, Ls : lobe secondaire. 18, Paramère droit. [Echelles $=0,5 \mathrm{~mm}$ (habitus); 0,1 $\mathrm{mm}$ (génitalias)]. pâle. Métatibias portant une pilosité jaune relativement raide, semi-dressée, de petits spinules noirs couchés et des épines brunes semi-dressées ou dressées. Tarses jaune pâle, rembrunis à l'apex (plus nettement sur les métatarses). Hémélytres brunrouge quasi unicolores porteuses d'une petite pilosité sétiforme blanche semi-dressée, relativement lâche, tout comme le scutellum. Incisure cunéale marquée. Membrane brun-jaunâtre unicolore. Face ventrale noire, à l'exception du xyphus du prosternum et des coxae qui sont jaune clair. Génitalias. Endophallus (fig. 15) restreint, portant apicalement un champ de denticules, se prolongeant au niveau du gonopore secondaire par deux lignes de denticules. Gonopore secondaire (G2) triangulaire et large. Ductus seminis (Ds) large. Paramère gauche (figs 16-17) falciforme, le lobe sensoriel (Ls) marqué, couvert de soies tout comme le corps. Bras non séparé de l'apophyse primaire, celle-ci large, se terminant assez abruptement (flèche). Paramère droit (fig. 18) massif, porteur de soies allongées.

Femelle similaire au mâle, brièvement redécrite par Carvalho \& Fontes (1972 : 528-529).

Discussion. Le spécimen guyanais d'Henicocnemis à notre disposition se caractérise à la fois par ses tibias antérieurs effilés et par la structure des génitalias du mâle, l'endophallus très simple faisant songer à la structure de Galapagomiris. Plus précisément, il se distingue de $H$. conspurcata Carvalho \& Gomes 1970a par l'absence des taches hémélytrales arrondies, jaunes, calleuses, caractéristiques de cette espèce, et par le pronotum unicolore. L'allongement des protibias, qui sont aplatis mais pas vraiment foliacés, permet d'écarter immédiatement $H$. alboornata Distant 1884 et $H$. patellata Stål 1860. Le pronotum et les hémélytres largement unicolores conduisent à rejeter $H$. boliviana Carvalho \& Fontes 1972 (dont le pronotum porte de larges bandes obliques et dont la corie est bicolore), H. fasciata Carvalho 1985a (dont l'endocorie porte une large bande claire), H. guttulosa Carvalho 1985a (dont le pronotum et les hémélytres sont tachés), $H$. peruviana Carvalho 1976 d et $H$. tucumanensis Berg 1883 (dont les pronota sont bicolores). H. carmelitana Carvalho 1985 a et $H$. castanea Carvalho 1985 a sont largement unicolores dorsalement. Ils se séparent en outre de notre Henicocnemis par leur endophallus plus complexe, comprenant une armature chitineuse sensu Chérot (2002, «ACH») et un spicule, ainsi que pour $H$. castanea par l'aspect dorsal mat. Enfin, $H$. albitarsis Stål 1862 se reconnait par son endophallus plus complexe, incluant deux processi cténoïdes et de plus nombreux lobes. Notre spécimen guyanais ne se rapproche guère que de $H$. luctuosa Stål 1860. Cette espèce reste mal connue (Carvalho \& Fontes 1972 : 529). Elle fut décrite du Brésil successivement par Stål (1860: 50) comme Deraeocoris luctuosus et par Poppius (1915: 38-39) comme Calocoris aterrimus, à chaque fois sur base d'une femelle (l'holotype par monotypie de D. luctuosus de Rio de Janeiro est préservé au Musée de Stockholm, celui de C. aterrimus, du Parana, l'étant au Musée de Gênes). Le mâle dont nous disposons correspond 
parfaitement tant à la description de Stål (op. cit.) qu’à celle de Poppius (op. cit.), descriptions dont il ne se sépare que par la taille légèrement inférieure (sachant cependant que la variabilité reste inconnue), par la couleur brune de l'angle interne de la grande cellule, concolore au reste de la nervure, par les taches du vertex et des yeux brunes, tranchant à peine avec le reste de la tête, et par la coloration du troisième article antennaire, plus nettement jaune. Compte tenu de la discussion ci-dessus, nous le considérons comme conspécifique du taxon de Stål (op. cit.) et non comme une espèce nouvelle («tibialis Carvalho» nom manuscrit), dont nous donnons une première description du mâle. Les deux spécimens argentins examinés s'avèrent plus foncés, pratiquement noirs sur la face dorsale, les taches céphaliques jaunes étant plus contrastées.

\section{Horciasinus signoreti (Stål 1859)}

Références. Capsus signoreti Stål 1859 : 257 (comme n. sp.) ; Horciasinus signoreti : Schuh 1995: 751 (catalogue); Carvalho et al. 2001: 148 (mention de Guyane française).

Spécimens examinés. Brésil : Amazonie : $1 \hat{\jmath}, 1 q$ : Manaus, 20.X.1963, G. Marlier leg. (FC ns 309-310). Novo Fiburgo : $1 \hat{\delta}$ : sans localité, Muller leg. (FC n $\left.{ }^{\circ} 311\right)$ (IRSNB). Guyane française : $2 \widehat{\jmath}$ : Kourou, environs de Cayenne, 27.IX.1979, J. Carayon leg. 2 우 : Patawa, point kilométrique 37, 11.IX.1998, A. Matocq leg. (MNHN).

Remarque. Espèce largement répandue en Amérique du Sud, connue d'Argentine, du Brésil, de Bolivie, de Colombie, du Guyana, du Paraguay, du Pérou, du Surinam, d'Uruguay et du Venezuela (Carvalho \& Jurberg 1974, 1976 ; Schuh 1995), mentionnée pour la première fois de Guyane française par Carvalho et al. (2001: 148).

\section{Horciasoides binigrus Carvalho, Costa $\&$ Chérot 2001}

Référence. Horciasoides binigrus Carvalho et al. 2001 : 149-151 (comme n. sp. décrite de Guyane française).

Remarque. Pas de donnée additionnelle disponible.

\section{Horciasoides gallicus Carvalho, Costa \& Chérot 2001}

Référence. Horciasoides gallicus Carvalho et al. 2001 : 151 (comme n. sp. décrite de Guyane française).

Remarque. Pas de donnée additionnelle disponible.

\section{Horciasoides nigridorsum Carvalho, Costa \& Chérot 2001}

Référence. Horciasoides nigridorsum Carvalho et al. 2001 : 151-153 (comme n. sp. décrite de Guyane française).

Remarque. Pas de donnée additionnelle disponible.

\section{Horciasoides punctatus (Carvalho 1976b)}

Références. Horcias (Horciasoides) punctatus Carvalho 1976b : 468-469 (comme n. sp.); Horcias punctatus: Schuh 1995 : 779 (catalogue).

Spécimens examinés. Brésil : Mato Grosso : Paratype : 19 : Sinop, Rio Teles Pires, IX.1979, Alvarenga \& Roppa (FC n ${ }^{\circ}$ 4477) (MNRJ). Guyane française : $1 q$ : Camopi - Oyapock, 19.XI.1969, mission Balachowsky - Gruner, octobre novembre 1969, piège lumineux (FC n ${ }^{\circ}$ 4618) (MNHN).

Remarque. Espèce nouvelle pour la Guyane française. Horciasoides punctatus fut décrit du Mato Grosso (Brésil).

\section{Iridopeplus pellucidipennis Bergroth 1910 (Figs 19-22)}

Références. Iridopeplus pellucidipennis Bergroth 1910: 61-63 (comme genre et espèce nouveaux décrits de Guyane française) ; Schuh 1995 : 785 (catalogue).

Spécimens examinés. Brésil : Amazonie : $6 \widehat{\jmath}$ 꾸우 : AM : $010 \mathrm{~km} \mathrm{31,} \mathrm{Embrapa,} \mathrm{02.X.1990,} \mathrm{02.I.1991,} \mathrm{18.VI.1991,}$ 03.IX.1991, L. P. Albuquerque \& J. E. Biada (FC n ${ }^{\circ}$ NE 7579) ; idem $\mathrm{km} \mathrm{244,} \mathrm{01.XI.1971,} \mathrm{B.} \mathrm{C.} \mathrm{Ratcliff} \mathrm{(FC} \mathrm{n}{ }^{\circ} \mathrm{NE}$ 83). 3 구 : Manaus, 29.V.1976, Eduardo coll., 14.V.1977, Joselita Lautos coll. et 16.V.1977, Rufino coll. (FC NE82, 84-85) (INPA). Guyana (République coopérative de Guyana, anciennement Guyane britannique) $: 2 \uparrow+q$ : au delà de Savane Kaieteur: High Forest 01 et 07.IX.1937 Richards \& Smart (FC ns 89-90) (BMNH). Guyane française : $1 \delta^{\Uparrow}$ : Route Nationale numéro 2, point kilométrique 62, 22.XI.1994, H. de Toulgoët \& J. Navatte (FC n $\left.{ }^{\circ} 1484\right)$. 2ふえ Nationale numéro 2, Piste Coralie, point kilométrique. 2,2, 01.XII.1994, A. Sénéceaux, H. de Toulgoët \& J. Navatte (FC ns 1485-1486) (MNHN).

Remarque. Iridopeplus pellucidipennis décrit de Guyane française fut ultérieurement signalé du Brésil et du Pérou (Carvalho \& Afonso 1977, ces auteurs décrivant les génitalias mâles). Les génitalias femelles n'étaient pas encore connus. Nous en fournissons cidessous une brève description.

Description des génitalias femelles. Anneaux pariétovaginaux (figs 19-20) grands, larges, leur marge antérieure proportionnellement la plus fine, convexe, entièrement couverte par une membrane (ou un repli du sac antérieur); leur marge postérieure concave; leur marge latéro-interne convexe, accolée à une plaque dorso-latérale en forme de massue et leur marge latéro-externe quasi droite, médianement étranglée. MiRs bien visible, pas de prolongation dorsale propre à l'anneau. Pas de plaque dorso-médiane. Une plaque ventrale, pratiquement limitée sous l'anneau, sa marge latéro-externe renforcée. Paroi postérieure (figs 21-22) avec une structure B (fig. 21) en 2 parties, formant un angle important, à savoir d'une part l'ensemble indivis "base + pied", le pied bifide et d'autre part le socle, dont les parts latérales sont fortement développées. Structures A grandes, plaques latérales bien marquées. Structures E grandes, couvrant partiellement les plaques latérales. Pas de structure $\mathrm{H}$. 


\section{Maxacalinus annulatus Carvalho, Costa \& Chérot 2001}

Référence. Maxacalinus annulatus Carvalho, Costa \& Chérot 2001 : 153154 (comme n. sp. décrite de Guyane française).

Remarque. Pas de donnée additionnelle disponible.

\section{Maxacalinus cuiabanus Carvalho 1976a}

Références. Maxacalinus cuiabanus Carvalho 1976a : 762 (comme n. sp.) ; Schuh 1995: 830 (catalogue).

Spécimens examinés. Guyane française : $1 \delta$ : Route Nationale numéro 2 vers Regina, 45 km S. de Cayenne, 31.V.1986, E.

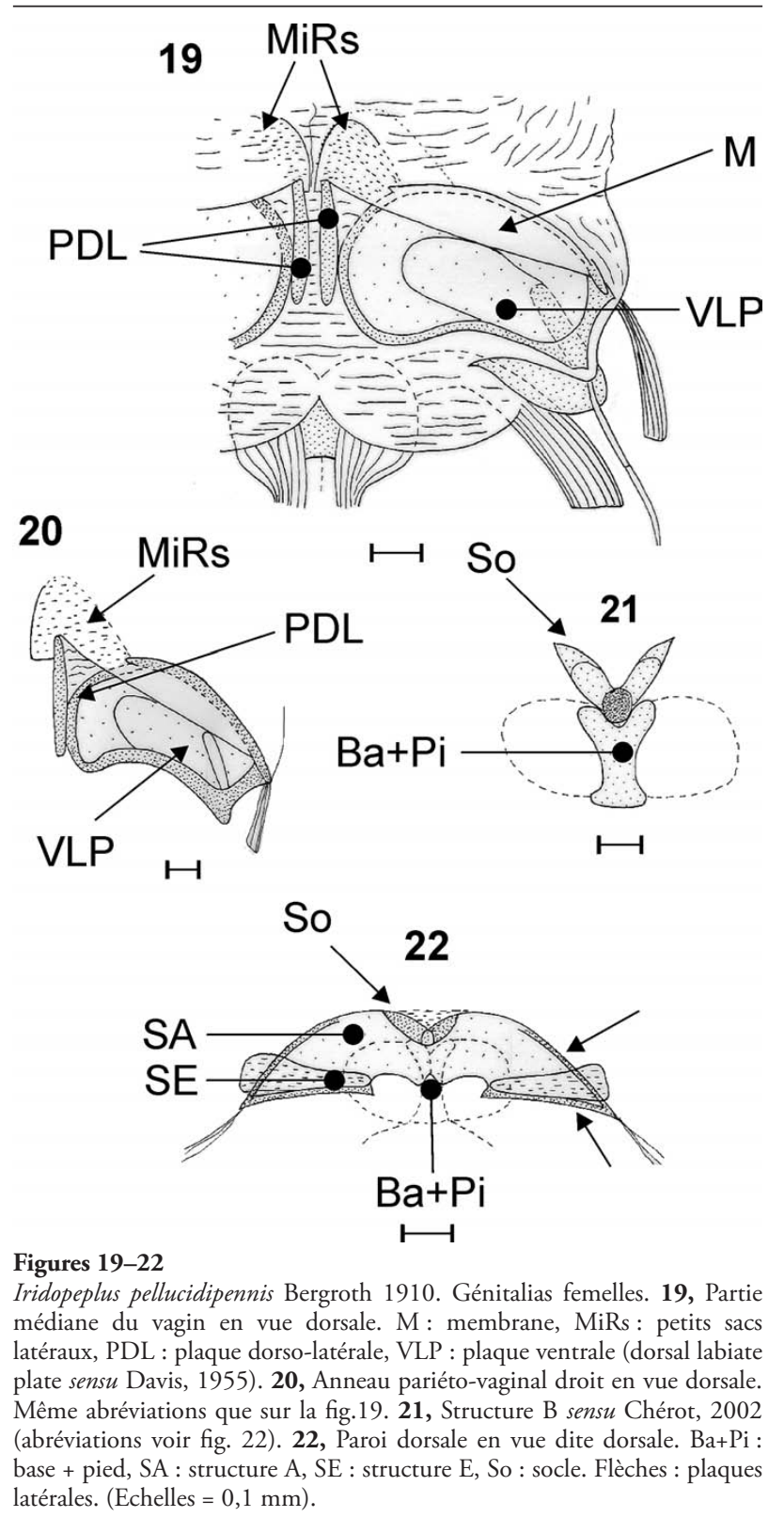

G. Riley \& D. A. Rider (FC n ${ }^{\circ} 1070$, identification J.C.M. Carvalho) (USNM). 1q : Embarcadère de Kaw, 01.X.2000, piège lumineux, D. Pluot-Sigwalt leg. (FC n4185). 1今': Patawa, 12.X.1998, A. Matocq leg. (FC n 4186) (MNHN).

Remarque. Espèce nouvelle pour la Guyane française, Maxacalinus cuiabanus fut décrit du Mato Grosso (Brésil).

\section{Maxacalinus hexapunctatus Carvalho, Costa \& Chérot 2001}

Référence. Maxacalinus hexapunctatus Carvalho, Costa \& Chérot 2001 : 154-157 (comme n. sp. décrite de Guyane française).

Remarque. Pas de donnée additionnelle disponible.

\section{Notholopus coreoides Carvalho 1975a}

Références. Notholopus coreoides Carvalho 1975a : 371 (comme n. sp.); Schuh 1995: 851 (catalogue).

Spécimens examinés. Brésil : Mato Grosso : Holotype $\overparen{\delta}$ Sinop, Rio Teles Pires, IX.1974, Alvarenga \& Roppa (MNRJ). Guyane française : $1 \hat{\jmath}, 290$ : Grand Santi, 23.IX.1999, A. Matocq leg. (FC n ${ }^{\circ}$ 4 4074, 4183-4184). 1 q : Patawa, 06.I.2002, (piège) malaise, J. Cerda leg. (FC n 4182). 19 : Camopi Oyapock, 19.XI.1969, mission Balachowsky - Gruner, octobre - novembre 1969, piège lumineux (MNHN).

Remarque. Espèce nouvelle pour la Guyane française, Notholopus coreoides fut décrite du Mato Grosso et est connue du Pérou (Carvalho \& Afonso 1977).

\section{Notholopus cuiabanus Carvalho 1975a}

Références. Notholopus cuiabanus Carvalho 1975a : 373-374 (comme n. sp.) ; Schuh 1995: 851 (catalogue).

Spécimens examinés. Brésil : Mato Grosso : Paratypes : 2 우 : Sinop, Rio Teles Pires, IX.1974, Alvarenga \& Roppa (MNRJ). Guyane française : $19:$ Grand Santi, 23.IX.1999, A. Matocq leg. (FC n²190) (MNHN).

Remarque. Espèce nouvelle pour la Guyane française. Comme l'espèce précédente, Notholopus cuiabanus fut décrite du Mato Grosso et est connue du Pérou (Carvalho \& Afonso 1977).

\section{Notholopus filicornis (Fabricius 1803)}

Références. Capsus filicornis Fabricius 1803: 245 (comme n. sp.); Paracalocoris lativentris Bergroth 1910 : 63-64 (comme n. sp. décrite de Guyane française, synonymie par Carvalho \& Gomes 1971 : 266) ; Notholopus filicornis Schuh 1995: 851 (catalogue).

Remarque. Espèce connue du Brésil, de Guyane française et du Surinam. Pas de données additionnelles disponibles.

\section{Phytocoris guianus n. sp. (Figs 23-26)}

Matériel type. Holotype $\delta$ : «French Guyana: Hwy (= Highway) N2 (= Nationale 2) to Regina, $45 \mathrm{~km} \mathrm{~s}$. of Cayenne 
(south), 31.V.1986, E. G. Riley \& D. A. Riden (USNM) [52²3'59”'W, 4³6'03”'N ; fig. 52, point 23].

Description. Mâle (holotype). Mensurations. Longueur totale : 3,4; largeur totale : 1,2. Longueur de la tête en vue dorsale : 0,2 ; largeur du diatone : 0,63 ; largeur du vertex : 0,21. Longueur des articles antennaires : I : 1,0 ; II : 1,9; III : 0,9 ; IV : 0,8. Longueur du pronotum : 0,5; largeur (postérieure) du pronotum : 1,1 . Longueur du cunéus : 0,7 ; largeur du cunéus : 0,4 . Coloration dorsale brun foncé avec des taches claires (fig. 23), jaunâtres ou rougeâtres, tête et pronotum jaunes tachés d'orange. Tête. Clypéus libre en vue dorsale, jaune, à peine taché d'orange au niveau du tylus; brides allongées, guttiformes incurvées, non carénées médianement, dépourvues de tubercule, jaunes, portant une tache longitudinale orangée ; joues dépourvues de tubercule, jaunes avec une ligne rouge-vin ; front allongé, quasi triangulaire en vue frontale, apparemment bombé (mais effondré médianement), jaunâtre légèrement taché d'orange ; pilosité du front et du vertex constituée de soies fines et relativement raides, dressées, de couleur noire ; yeux en contact avec le bord antérieur du pronotum, occupant environ, en vues frontale et latérale, les $2 / 3$ de la hauteur de la tête ; yeux jaune foncé tachés de brun orangé et de rouge, apparemment glabres; fossettes antennifères jaunes avec une petite tache rouge ; premier article antennaire allongé, épaissi sub-basalement et apicalement, étranglé médianement, jaune taché de rouge, portant de courtes soies semi-dressées ou couchées, blanches et quelques soies raides, spiniformes, dressées, noires ; seconde article allongé, jaune avec une étroite ligne noire sur quasi toute sa longueur ; articles III jaune basalement et apicalement, noir médianement ; article IV noir ; vertex bien délimité du front, très finement marginé ; marge du vertex rouge-vin ; surface du vertex finement sillonnée médianement de sa marge à la limite du front, de forme complexe, en vue frontale chaque moitié subtrapézoïdale ; rostre rouge-brun, localement taché de jaune, atteignant le pygophore. Pronotum. Anneau apical jaune orangé, sa pilosité sétiforme dressée, relativement raide, allongée, de couleur noire et sétiforme à soyeuse, courte, couchée, argentée sous lumière incidente ; callosités nettement délimitées, jaunes tachées d'orange, portant une pilosité identique à celle du disque, ne joignant pas les bords latéraux du pronotum ; angles antérieurs du pronotum chacun porteur d'une très longue soie sétiforme dressée; disque jaune, taché d'orange clair ; pilosité du disque sétiforme dressée, relativement raide, allongée (plus longue sur ses marges latérales), de couleur noire et sétiforme à soyeuse, courte, couchée ou semi-dressée, argentée sous lumière incidente (cette dernière restreinte aux marges latérales) ; bord postérieur du pronotum quasi droit médianement, faiblement incurvé concave latéralement, angles huméraux arrondis. Pas de "karinal knob». Scutellum quasi plan, jaune clair basalement, jaune portant de petites taches rouge foncé apicalement ; sa pilosité double, constituée de soies sétiformes noires, semi-dressées, et de soies laineuses blanches couchées, ces dernières rares ; mésoscutum largement couvert (probablement est-ce un artéfact dû à l'état du spécimen), jaune foncé, ses fossettes latérales peu visibles, jaunes. Pattes très allongées ; mésofémurs orange-brun, tachés de jaune, les taches arrondies ; métafémurs de type Phytocoris c.-à-d. rainuré et allant en s'amincissant de la base vers l'apex, jaune taché de rouge dorsalement, portant une courte pilosité, semi-dressée, plus ou moins raide ; métatibias fins, très allongés, jaunes tachés de rouge, portant une pilosité similaire à celle des métafémurs, de larges épines noires et des spinules de la même couleur ; tarses bruns. Hémélytres. Pilosité hémélytrale double, constituée de soies noires, relativement souples, semi-dressées ainsi que de soies blanches, nettement soyeuses, couchées. Coloration jaune taché de brun, la base de chaque soie noire dans une petite tache rouge foncée ; clavii avec quelques soies noires, dressées, raides à l'apex, basalement et apicalement jaunes, médianement largement tachés de brun, tout comme l'endocorie; exocories jaunes porteuses de taches oblongues rouge claire; endocories et parascutum porteurs de taches rouge-vin ; cunei jaunes tachés de rouge clair et de rouge-vin, particulièrement à leur apex ; membrane claire, maculée de brun, portant une tache jaunâtre sous l'apex des cunei et des petites cellules; les nervures rouges foncées, le rebord de la grande cellule à la limite de la petite jaune. Face ventrale jaune, coxae et abdomen clairs; pleures jaunes ou (sur le thorax) jaunes tachés d'orange. Génitalias. Endophallus (fig. 24) très simple, portant deux petits champs de denticules (flèches). Pas d'ACH, ni de spicule, ni de peigne. Gonopore secondaire (G2) large, dépourvu de sclérite. Ductus

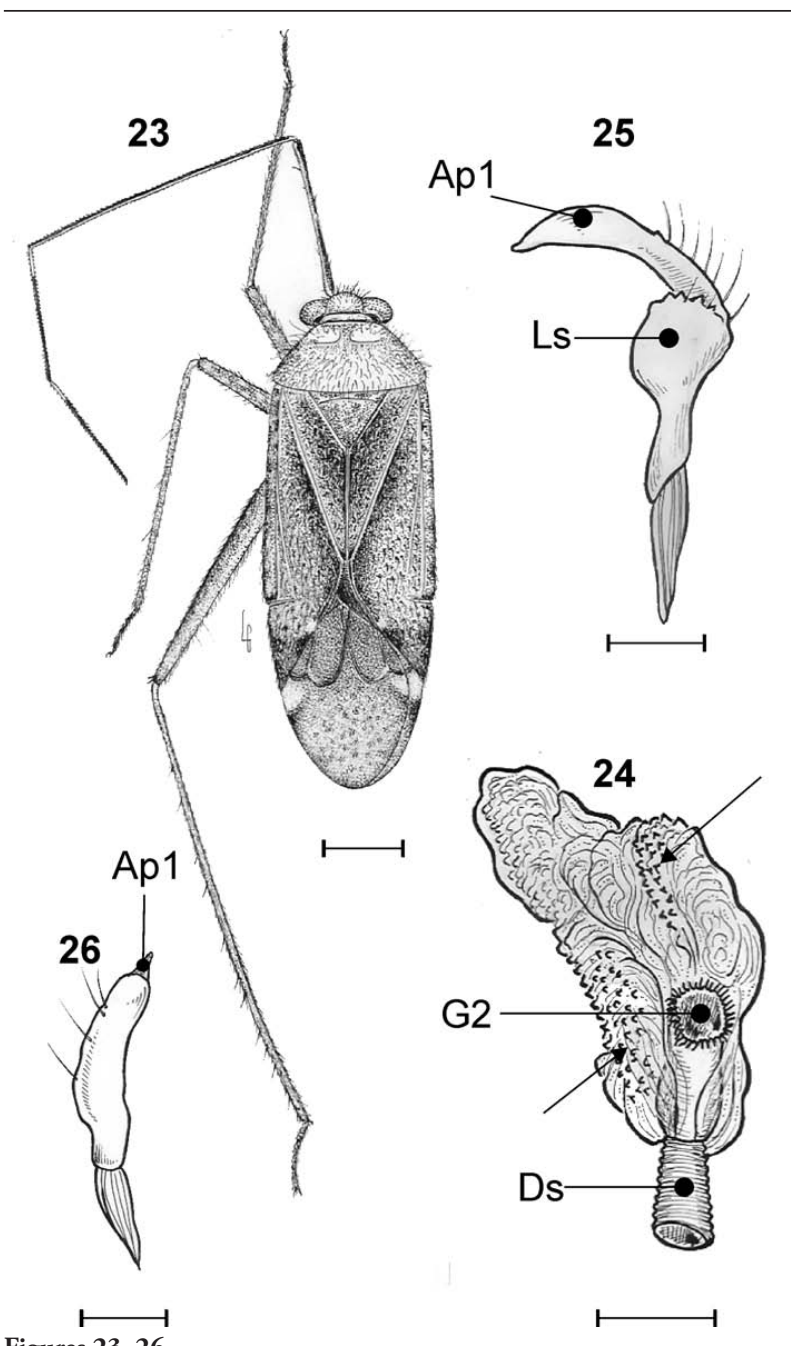

Figures 23-26

Phytocoris guianus n. sp. 23, Habitus en vue dorsale. 24, Endophallus en vue latéro-dorsale. Ds : ductus seminis, G2 : gonopore secondaire. Flèches : champs de denticules. 25, Paramère gauche en vue dorsale. Ap1 : apophyse primaire, Ls: lobe sensoriel. 26, Paramère droit en vue dorsale. Ap1 : apophyse primaire. [Echelle $=0,5 \mathrm{~mm}$ (habitus) ; 0,1 $\mathrm{mm}$ (génitalias)]. 
seminis (Ds) large. Paramère gauche (fig. 25) fortement courbé au niveau du corps, son lobe sensoriel (Ls) large, denticulé sur sa marge postérieure ; bras et apophyse primaire non nettement séparés, l'apophyse primaire (Ap1) allongée. Paramère droit (fig. 26) classique, le lobe sensoriel (Ls) très restreint. Apophyse primaire (Ap1) allongée, en pointe émoussée, nettement séparée du bras.

Femelle inconnue.

Derivatio nominis. L'épithète réfère au département de Guyane française dans lequel la nouvelle espèce fut collectée.

Commentaire. Parmi les Phytocoris spp. centre- et sud-américains dépourvus de soie squamiforme, à hémélytres sans tache claire ronde ou ovale et de longueur totale inférieure à $8 \mathrm{~mm}$, Phytocoris guianus n. sp. se caractérise par son habitus, en particulier sa petite taille $(3,4 \mathrm{~mm})$, par la structure très simple de son endophallus, dépourvu d'ACH, de peigne, de spicule, de palettes en spirale (contrairement à P. cylapinus Carvalho \& Gomes 1970b) ou de lobe spiculiforme, et par son paramère gauche dépourvu d'apophyse secondaire (contrairement notamment à P. citrinoides Carvalho \& Gomes 1970b) avec un lobe sensoriel grand, denticulé sur sa marge postérieure.

\section{Phytocoris jugatus n. sp. (Figs 27-30)}

Matériel type. Holotype $\widehat{\delta}$ : «French Guyana : Montagne des Singes, n(ea)r Kourou, 3.VI.1986, E. G. Riley \& D. A. Rider» (USNM) [52 41'26”W, 5 03'33”N si on prend la piste bordant à l'est la Montagne des Singes à hauteur de Sokoumou ; fig. 52, point 14]. Paratypes : 19 : mêmes indications que l'holotype $(\mathrm{MNRJ}) ; 1$ đo et 3 우 : «French Guyana : Hwy (= Highway) N2 (= Nationale 2) to Regina, $45 \mathrm{~km} \mathrm{~S}$ (= South) of Cayenne, 31.V.1986, E. G. Riley \& D. A. Rider»; $1 \delta$ : «French Guyana : $13 \mathrm{~km} \mathrm{SSE}$ of St. Laurent (= Saint Laurent), 8.VI.1986, E. G. Riley \& D. A. Rider» (MNRJ) [tous collectés à la lampe à vapeur] ; 1 : «Guyane Française : Patawa, malaise, 6.I.2002, J. Cerda leg.»; $1 \delta^{\lambda}$ : "Guyane Française : Acarounay, Auberge du Bois Diable, 28.IX.2000, A. Matocq leg. "; 1 : : «Guyane Française : Patawa Pk 37, 12.IX.1988, A. Matocq leg.» ; 2 9 : "Guyane Française: Kaw, Patawa Pk 37,5, 29.IX.2000, A. Matocq leg.»(MNHN).

Description. Mâle (holotype, paratypes). Mensurations (holotype). Longueur totale : 4,0 ; largeur totale : 1,6. Longueur de la tête en vue dorsale : 0,2; largeur du diatone : 0,9; largeur du vertex : 0,3 . Longueur des articles antennaires : I : 0,8 ; II : 2,2 ; III : 1,2; IV : 1,0. Longueur du pronotum : 0,6 ; largeur (postérieure) du pronotum : 1,3. Longueur du cunéus : 0,7; largeur du cunéus : 0,5 . Coloration dorsale brun foncé avec des taches claires (fig. 27), jaunâtres, brun clair ou même verdâtres. Tête. Clypéus libre en vue dorsale, jaune avec une marque longitudinale rouge-vin de forme complexe, faisant songer à un chandelier à base élargie, à bras médian raccourci et aux bras latéraux allongés et un anneau basal de la même couleur (parfois il s'agit de deux bandes latérales se dirigeant l'une vers l'autre mais ne se rejoignant pas) ; brides non carénées médianement, dépourvues de tubercule, bordées de jaune, portant une tache longitudinale brune plus ou moins développée; joues dépourvues de tubercule, jaunes bordées de rouge-vin ; front légèrement bombé, finement strié, jaunâtre à verdâtre selon les spécimens, portant latéralement deux bandes brunes, rosées ou rougeâtres, lesquelles se poursuivent et se croisent sur le vertex (sur un paratype femelle du MNHN, ces bandes ne se poursuivent pas sur le vertex), où elles sont incurvées, ces bandes délimitant une série de 4 zones jaunes claires de forme vaguement triangulaire; pilosité du front et du vertex complexe, constituée de différentes sortes de soies, à savoir des soies fines et relativement raides, dressées, de couleur noire, d'autres similaires apparaissant argentée sous lumière incidente, et enfin des soies couchées, plus nettement soyeuses, argentées; yeux en contact avec le bord antérieur du pronotum, atteignant la marge ventrale de la tête, de couleur variable, allant de brun orangé à brun foncé ou rougeâtre (holotype) au milieu, plus clairs latéralement, apparemment glabres; premier article antennaire allongé, épaissi sub-basalement et apicalement, étranglé médianement, brun foncé taché de jaune, portant de courtes soies semi-dressées et quelques soies raides, jaunes (paratype MNRJ) ou noires (holotype) dressées; second article allongé, brunâtre, avec un anneau basal jaune et un anneau médian brun foncé (parfois ce dernier anneau effacé ou plus clair que le reste de l'article); articles III et IV brun foncé avec un anneau basal jaune ; vertex immarginé mais porteur d'un bourrelet apical qui pourrait faire songer à une marge ; rostre rouge-brun, atteignant quasi l'apex de l'abdomen. Pronotum. Anneau apical jaunâtre, taché de rouge ou de brun, sa pilosité sétiforme dressée, relativement raide, allongée, de couleur noire et sétiforme à soyeuse, courte, couchée, argentée sous lumière incidente; callosités plus ou moins nettement délimitées selon les individus, brunes, portant une pilosité identique à celle du disque, ne joignant pas les bords latéraux du pronotum; disque brun, taché de jaune clair, rarement de verdâtre, portant trois bandes longitudinales jaunâtres ou verdâtres: une médiane atteignant la marge antérieure, et deux latérales, derrière les callosités, incurvées, rejoignant antérieurement la bande médiane ; pilosité du disque sétiforme dressée, relativement raide, allongée (plus longue sur ses marges latérales, cependant même là plus courte et moins raide que sur l'anneau apical), de couleur noire et sétiforme à soyeuse, courte, couchée ou semi-dressée, argentée sous lumière incidente; bord postérieur du pronotum quasi droit médianement, faiblement incurvé concave latéralement, bordé de jaune, particulièrement au niveau des angles huméraux, qui sont arrondis. Pas de «karinal knob». Scutellum jaune clair, portant la même pilosité que le pronotum, en légèrement plus fin ; mésoscutum largement couvert, jaune-orangé, ses fossettes latérales orangées tachées de brun. Pattes. Fémurs tachés, tibias avec deux anneaux pâles, un médian et un apical, et de longues épines brun-rouge; spinules présents, noirs ; tarses jaunâtres. Hémélytres. Pilosité hémélytrale relativement complexe, constituée de soies noires, relativement souples, semi-dressées, de soies blanches, relativement souples, dressées, ainsi que de soies couchées, blanches, soyeuses, particulièrement nombreuses à la base des clavii et des coria, pratiquement absentes sur les cunei. Clavii jaunes à verdâtres tachés de brun et de clair, les taches les plus marquées au milieu et à l'extrême apex, la tache apicale portant une petite touffe de courtes soies noires, raides, dressées ; endocoria jaunes ou bruns avec trois taches noires, une sub-basale et deux apicales, toutes plus ou moins étendues ou foncées selon les spécimens; exocoria jaunes ou bruns, avec plusieurs petites taches oblongues plus foncées sur les marges; cunei jaunes ou bruns, leur apex plus foncé, portant deux petites taches brunes ou noires sur leur marge interne, chacune avec une courte touffe 
de soies noires, raides, dressées ; membrane foncée. Face ventrale brun-rougâtre, coxae et abdomen clairs. Génitalias. Endophallus (fig. 30) comprenant un grand peigne (Pe) portant 8 larges dents ainsi qu'un petit champ de denticules. Pas d'ACH, ni de spicule. Gonopore secondaire (G2) large, dépourvu de sclérite. Ductus seminis (Ds) large. Paramère gauche (fig. 28) fortement courbé au niveau du corps, son lobe sensoriel (Ls) digitiforme allongé, dressé vers le bras; bras et apophyse primaire non nettement séparés, l'apophyse primaire (Ap1) en pointe allongée. Paramère droit (fig. 29) particulièrement développé, le lobe sensoriel (Ls) large, digitiforme, dressé, dépassant en vue dorsale l'apophyse primaire. Apophyse primaire (Ap1) allongée, en pointe émoussée, non nettement séparée du bras.

Femelle (paratypes) similaire au mâle, leurs génitalias inconnus.

Derivatio nominis. L'épithète réfere au joues jaunes bordées de rouge-vin de la nouvelle espèce.

Commentaire. Parmi les Phytocoris spp. centre- et sud-américains dépourvus de soie squamiforme, à hémélytres sans tache claire ronde ou ovale et de longueur totale inférieure à $8 \mathrm{~mm}$, Phytocoris jugatus n. sp. se caractérise par son habitus, en particulier sa taille relativement petite $(4 \mathrm{~mm})$, par la structure de son endophallus, dépourvu d'ACH, de spicule, de palettes en spirale ou de lobe spiculiforme mais comprenant un large peigne à 8 dents, et par la structure de ses deux paramères, singulièrement celle de leur lobe sensoriel digitiforme. Les trois espèces connues du genre Gracilamiris Stonedahl \& Henry 1991, décrites des Etats-Unis (Arizona, Floride, Mississipi, Maryland, Texas), se caractérisent, outre leur habitus allongé, par un lobe sensoriel du paramère gauche portant une prolongation incurvée et denticulée. Elles se différencient de $P$. jugatus n. sp. par de nombreux caractères, notamment, au niveau des génitalias, par l'absence de peigne phallique, la présence d'un spicule, une prolongation du paramère gauche beaucoup plus complexe, un paramère droit nettement différent du gauche et lui-même assez complexe, etc... Les relations éventuelles de ces curieux Mirini et de P. jugatus restent à établir dans le cadre beaucoup plus général d'une clarification du complexe Phytocoris.

\section{Poeas castanea Carvalho, Costa $\&$ Chérot 2001}

Référence. Poeas castanea Carvalho, Costa \& Chérot 2001: 160-161 (comme n. sp. décrite de Guyane française).

Remarque. Pas de donnée additionnelle disponible.

\section{Taedia bananalensis Carvalho \& Costa 1993}

Référence. Taedia bananalensis Carvalho \& Costa 1993 : 221-222 (comme n. sp.).

Spécimens examinés. Brésil : Goias : Paratype : $1 q$ : île de Bananal, Santa Isabel do Moro, VI.1961, M. Alvarenga leg. (FC $\mathrm{n}^{\circ}$ 4370) (MNRJ).Guyane française : $1 \delta$ : Forêt de Balate, St Jean du Maroni, 16.X.1969, mission Balachowsky - Gruner, octobre - novembre 1969 (FC n ${ }^{\circ}$ 4654) (MNHN).

Remarque. Taedia bananalensis n'avait pas encore été signalé de Guyane française. Cette espèce fut décrite de Goias (Brésil), sur la base d'un seul exemplaire de chaque sexe. Le phallus de notre spécimen est très similaire à celui représenté par Carvalho \& Costa (op. cit., fig. 2), ses deux spiculi étant cependant proportionnellement plus allongés que sur l'holotype.

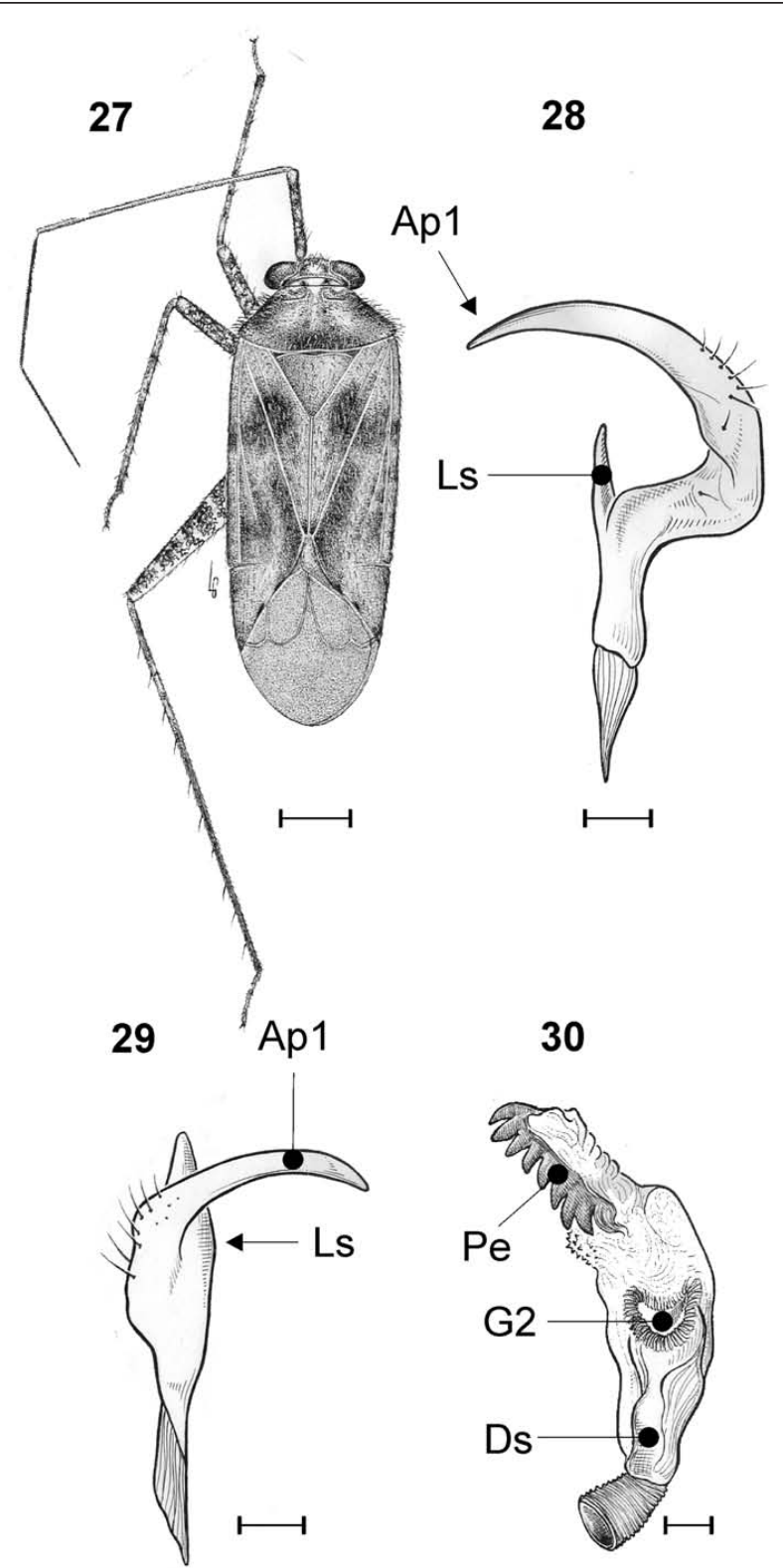

Figures 27-30

Phytocoris jugatus n. sp. 27, Habitus en vue dorsale. 28, Paramère gauche en vue dorsale. Ap1 : apophyse primaire, Ls : lobe sensoriel. 29, Paramère droit en vue dorsale. Ap1 : apophyse primaire, Ls : lobe sensoriel. 30, Endophallus en vue latéro-dorsale. Ds : ductus seminis, G2 : gonopore secondaire, Pe : peigne. [Echelles $=0,5 \mathrm{~mm}$ (habitus); 0,1 $\mathrm{mm}$ (génitalias)]. 


\section{Taedia bilutea Carvalho, Costa \& Chérot 2001}

Référence. Taedia bilutea Carvalho, Costa \& Chérot 2001: 161-162 (comme n. sp. décrite de Guyane française).

Remarque. Pas de donnée additionnelle disponible.

\section{Taedia cajabiana Carvalho $1976 \mathrm{c}$}

Références. Taedia cajabiana Carvalho 1976c : 769-770 (comme n. sp.); Schuh 1995: 952 (catalogue).

Spécimens examinés. Brésil : Mato Grosso : Holotype $\widehat{\jmath}$ : Sinop, Rio Teles Pires, 09.II.1974, Alvarenga \& Roppa (MNRJ). Guyane française : $1{ }^{\Uparrow}$ : Massikiri - Oyapock, 18.XI.1969, mission Balachowsky - Gruner, octobre - novembre 1969, piège lumineux. (FC n 4655) (MNHN).

Remarque. Espèce nouvelle pour la Guyane française, Taedia cajabiana fut décrite du Mato Grosso. Les génitalias denotre spécimen mâlede Guyane concordent parfaitement aux figures 15 à 18 de Carvalho (op. cit.), en particulier l'ACH complexe.

\section{Taedia coimbrai Carvalho, 1975c}

Références. Taedia coimbrai Carvalho 1975c : 505-506 (comme n. sp.); Schuh 1995 : 953 (catalogue).

Spécimens examinés. Guyane française : $1 \hat{\jmath}$ : Massikiri - Oyapock, 18.XI.1969, mission Balachowsky - Gruner, octobre - novembre 1969, piège lumineux. (FC n $\left.{ }^{\circ} 4627\right) .1 \%$ : Acournay, Auberge du Bois Diable, 28.IX.2000, A. Matocq leg. (FC n ${ }^{\circ}$ 4191) (MNHN).

Remarque. Espèce nouvelle pour la Guyane française, Taedia coimbrai fut, elle aussi, décrite du Mato Grosso.

\section{Taedia compactoides Carvalho 1975b}

Références. Taedia compactoides Carvalho 1975b : 190-192 (comme n. sp.); Schuh 1995 : 953 (catalogue).

Spécimens examinés. Brésil : Mato Grosso : Holotype $\widehat{\jmath}$ : Sinop, Rio Teles Pires, IX.1974, Alvarenga \& Roppa (MNRJ). Guyane française : $1 q$ : Route de la Conté, 01.XII.1969, mission Balachowsky - Gruner, octobre - novembre 1969, piège lumineux (FC n ${ }^{\circ}$ 4624). 2 우 : Massikiri - Oyapock, 17-18.XI.1969, mission Balachowsky - Gruner, octobre - novembre 1969, piège lumineux. (FC ns 4625-4626) (MNHN).

Remarque. Espèce nouvelle pour la Guyane française, Taedia compactoides fut décrite du Mato Grosso et est connue du Pérou (Carvalho \& Afonso 1977).

\section{Taedia pauwelsi Carvalho, Costa \& Chérot 2001}

Références. Taedia pauwelsi Carvalho, Costa \& Chérot 2001 : 162-164 (comme n. sp. décrite de Guyane française).

Remarque. Pas de donnée additionnelle disponible.

\section{Taedia semilota (Stål 1860)}

Références. Capsus semilota Stål 1860 : 50 (comme n. sp.); Adelphocoris umbratus Bergroth 1910: 64-65 (comme n. sp. décrite de Guyane française ; synonymie par Carvalho \& Gomes 1971 : 277); Taedia semilota : Schuh 1995 : 957 (catalogue).

Remarque. Pas de donnée additionnelle disponible.

\section{Taedia signata Carvalho \& Gomes 1971}

Références. Taedia signata Carvalho \& Gomes 1971 : 277 (comme n. sp.) ; Schuh 1995 : 957 (catalogue); Carvalho, Costa \& Chérot 2001: 148 (mention de Guyane française).

Spécimens examinés. Brésil : Minas Gerais : Holotype $\widehat{\partial}$ : 1947, C.R. Claro (MNRJ). Guyane française : 1 : Massikiri - Oyapock, 27.XI.1969, mission Balachowsky - Gruner octobre - novembre 1969, piège lumineux (FC n 4619). 1 웅 (?) : Alicoyo - Oyapock, 13.XI.1969, mission Balachowsky - Gruner octobre - novembre 1969, piège lumineux (FC $\mathrm{n}^{\circ}$ 4620). 1 ㅇ (?) : Itani, Île de Touenké, 19-21.XI.1975, M. Boulard, P. Jauffret \& P. Pompanon leg. (FC n 4621). 1ठ, 2우: Patawa, point kilométrique 37, 11-12.IX.1998, A. Matocq leg. (FC n's 4152-4153, 4189). 1ठ: Patawa, point kilométrique 37,5, 29.IX.2000, A. Matocq leg. (FC n 4623). Surinam : 19 : Itani, Carbet Lavaud, rive surinamienne, 0304.XII.1975, M. Boulard, P. Jauffret \& P. Pompanon leg. (FC $\mathrm{n}^{\circ}$ 4622) (MNHN).

Remarque. Taedia signata fut décrit par Carvalho \& Gomes (op. cit.) du Minas Gerais et avait été signalé de Guyane française par Carvalho et al. (2001) sans indication de localité.

\section{Taedia xinguana Carvalho 1975b}

Références. Taedia xinguana Carvalho 1975b : 204-206 (comme n. sp.); Schuh 1995 : 959 (catalogue).

Spécimens examinés. 3qq: Grand Santi, fin.IX.1999, D. Pluot-Sigwalt leg. (FC n4197-4199) (MNHN).

Remarque. Espèce nouvelle pour la guyane française, Taedia xinguana fut décrite par Carvalho (op. cit.) du Mato Grosso, Xingu.

\section{Resthenini}

\section{Prepops poppii (Bergroth 1910)}

Références. Platytylellus poppii Bergroth 1910 : 60 (comme n. sp. décrite de Guyane française) ; Prepops poppii Carvalho 1959 : 340 (nov. comb. in catalogue); Schuh 1995: 995 (catalogue).

Remarque. Pas de donnée additionnelle disponible.

\section{Prepops rollei (Reuter 1910)}

Références. Platytylellus rollei Reuter 1910: 29 (comme n. sp.); Prepops rollei : Schuh 1995 : $995-996$ (catalogue); Carvalho, Costa \& Chérot $2001: 148$.

Remarque. Pas de donnée additionnelle disponible. 


\section{Orthotylinae}

\section{Orthotylini}

\section{Guianocoris n. g.}

Description. Mâle (holotype). Habitus. Corps allongé, lisse, unicolore, revêtu de soies couchées, épaisses, blanches, et de soies érigées noires plus fines (fig. 31). Clypéus arrondi, saillant et comprimé. Yeux contigus au pronotum, grands. Joues et brides petites. Bord postérieur du pronotum non incurvé médianement, les angles huméraux arrondis, les callosités invisibles, remplacées par un bourrelet antérieur de couleur blanc ivoire, ou réduites, s'ajoutant au bourrelet précité. Mésoscutum largement couvert, scutellum plan. Hémélytres allongés, embolium bien marqué. Césure embolio-coriale en $S$ allongé. Cunéus plus long que large, mal séparé du paracunéus.

Espèce-type. Guianocoris unicolor n. sp.

Derivatio nominis. Le nom du nouveau genre réfère au département de Guyane française dans lequel ce taxon fut collecté. Genre grammatical: masculin [Guianocoris «est un nom composé» au sens du glossaire du Code International de Nomenclature Zoologique (Commission Internationale de Nomenclature Zoologique, 1999), nom qui se termine en un mot grec ( $\chi o ́ \varrho \iota \varsigma$, punaise) translittéré en latin et grammaticalement masculin. En vertu de l'Article 30.1.2 du Code (op. cit.), Guianocoris est donc grammaticalement masculin].

Commentaire. Parmi les Orthotylini centre- et sudaméricains, Guianocoris n. g. se distingue :

(1) par la largeur "restreinte» de ses deux derniers articles antennaires, inférieure à celle des deux premiers articles antennaires et de plus non fusiformes ;

(2) par sa surface dorsale très finement ponctuée [cette ponctuation trop petite pour correspondre à ce que Carvalho (1985b : 250) entend par «Pronotum ou hémélytre distinctement ponctués» dans le couplet 4 de sa clé des Orthotylini néotropicaux], dépourvue de soies squamiformes ;

(3) par ses yeux accolés au bord antérieur du pronotum ;

(4) par sa coloration jaune-verdâtre et son premier article antennaire épais, court ;

(5) par son vertex apparemment bombé, portant un bourrelet postérieur et non pas une carène ;

(6) par les marges latérales de ses hémélytres subparallèles et par la fracture cunéale normale ;

(7) par le corps pileux ;

(8) par le clypéus arrondi, saillant et comprimé.

La possession conjointe de ces états de caractères conduit au couplet 43 de la clé de Carvalho (op. cit.) et au genre Cyrtotylus Bergroth 1922, nonobstant le fait que la longueur du rostre - utilisée au couplet 39 - de notre spécimen est inconnue [apparemment le rostre - englué - dépasserait les coxae antérieurs, quoiqu'il en soit la pilosité dorsale exclut Rhinocapsidea Reuter 1908 qui est glabre, et ni l'habitus, ni les génitalias ne correspondent à Linharesmiris Carvalho 1986 (A tort repris comme "Linharesmiris Carvalho 1984" dans le couplet 40 de la clé de Carvalho (1985b: 252)]. Ainsi que nous le discuterons ci-dessous après la description spécifique, le genre Cyrtotylus Bergroth 1922, tel qu'actuellement défini dans la littérature sur base de ses membres, apparaît inhomogène et pourrait fort bien être un groupe polyphylétique. Selon nous, Guianocoris unicolor se sépare suffisamment nettement de Cyrtotylus rubricatus Bergroth 1922, espèce-type du genre Cyrtotylus, pour justifier la création d'un nouveau genre.

\section{Guianocoris unicolor $\mathrm{n}$. sp. (Figs 31-35)}

Matériel type. Guianocoris unicolor: Holotype $\widehat{\sigma}$ : «French Guyana: Hwy (= Highway) N2 (= Nationale 2) to Regina, $67 \mathrm{~km}$ of Cayenne, 4.VI.1986, E. G. Riley \& D. A. Rider» (USNM) [52 $19^{\prime} 37^{\prime \prime} \mathrm{W}, 4^{\circ} 25^{\prime} 48^{\prime \prime} \mathrm{N}$; fig. 52, point 25]. Nota : spécimen étiqueté par Carvalho comme holotype de "Guianerius unicolor n. g., n. sp.», un binôme manuscrit dont la partie de niveau genre ne saurait être rendue disponible sans générer un homonyme plus récent de Guianerius Distant 1903, un Mirinae de Malaisie.

Autres taxa examinés pour comparaison: Cyrtotylus ruber Carvalho \& Carpintero 1991a: Argentine: Salta:

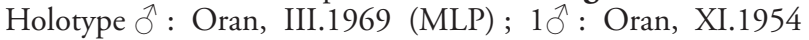
(CAR). Cyrtotylus rubricatus Bergroth 1922 : Pérou : Ucayali :

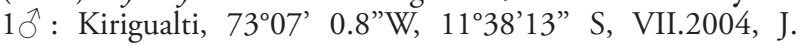
Williams (CAR). Cyrtotylus wygodzinskyi Carvalho 1950: Holotype $\hat{O}$ : Argentine : Tucuman : 21.X.1948, Wygodzinski coll. (MLP) ; Argentine : Cordoba : 19 : Cruz del Ege, II.1999, Barriga (CAR). Cyrtotylus sp. A (groupe de C. wygodzinskyi) : Argentine : Santiago Del Estro : 10 , $2 q \uparrow$ : Añatuya, XI.1998, Carpintero (CAR). Cyrtotylus cruciatus Carvalho \& Carpintero 1991b (groupe de C. wygodzinskyi): Argentine: Santiago Del Estro : $2 \widehat{ึ}$ : Añatuya, XI.1998, Carpintero (CAR). Cyrtotylus sp. D (groupe de C. catarinensis) : Pérou : Cuzco : 1ठ: Cashiriari, 04-22.VII.2005, à la lumière, Williams, J. (CAR). Cyrtotylus sp. E (groupe de C. catarinensis) : Pérou : Ucayali : $10^{\Uparrow}$ : Kirigualti, $73^{\circ} 07^{\prime} 0.8^{\prime}$ W, $11^{\circ} 38^{\prime} 13^{\prime \prime S}$, VII.2004, J. Williams (CAR).

Description. Mâle (holotype). Mensurations. Longueur totale : 2,8 ; largeur totale : 1,0 . Longueur de la tête en vue dorsale : 0,1 ; largeur du diatone : 0,6 ; largeur du vertex : 0,2. Longueur des articles antennaires : I : 0,2 ; II : 1,0 ; III : 0,3 ; IV : 0,2. Longueur du pronotum : 0,4; largeur (postérieure) du pronotum : 1,0. Longueur du cunéus : 0,9 ; largeur du cunéus : 0,4. Coloration générale. Coloration dorsale jaune pâle, membrane foncée. Tête. Clypéus libre, non couvert par le front en vue dorsale, jaune orangé unicolore, arrondi, saillant et comprimé. Brides et joues petites, jaunes pâles unicolores, ni carénées, ni tuberculées. Front apparemment lisse (malheureusement affaissé médianement sur l'holotype), portant une pilosité sétiforme blanche, dressée. Yeux grands, pileux, rouge-brun, occupant en vue frontale près des $3 / 4$ de la hauteur de la tête et en vue latérale toute cette hauteur, directement en contact avec le bord antérieur du pronotum. 
Premier article antennaire jaune brunâtre, court et épais, 5 fois plus court et légèrement plus épais que le second, revêtu de courtes soies blanches, leur longueur inférieure à la largeur de l'article (contrairement aux soies érigées des articles III et IV). Deuxième article antennaire allongé, de même couleur que le premier, revêtu de soies blanches semi-dressées de longueur voisines de la largeur de l'article et de soies noires beaucoup plus courtes. Troisième et quatrième articles antennaires de même
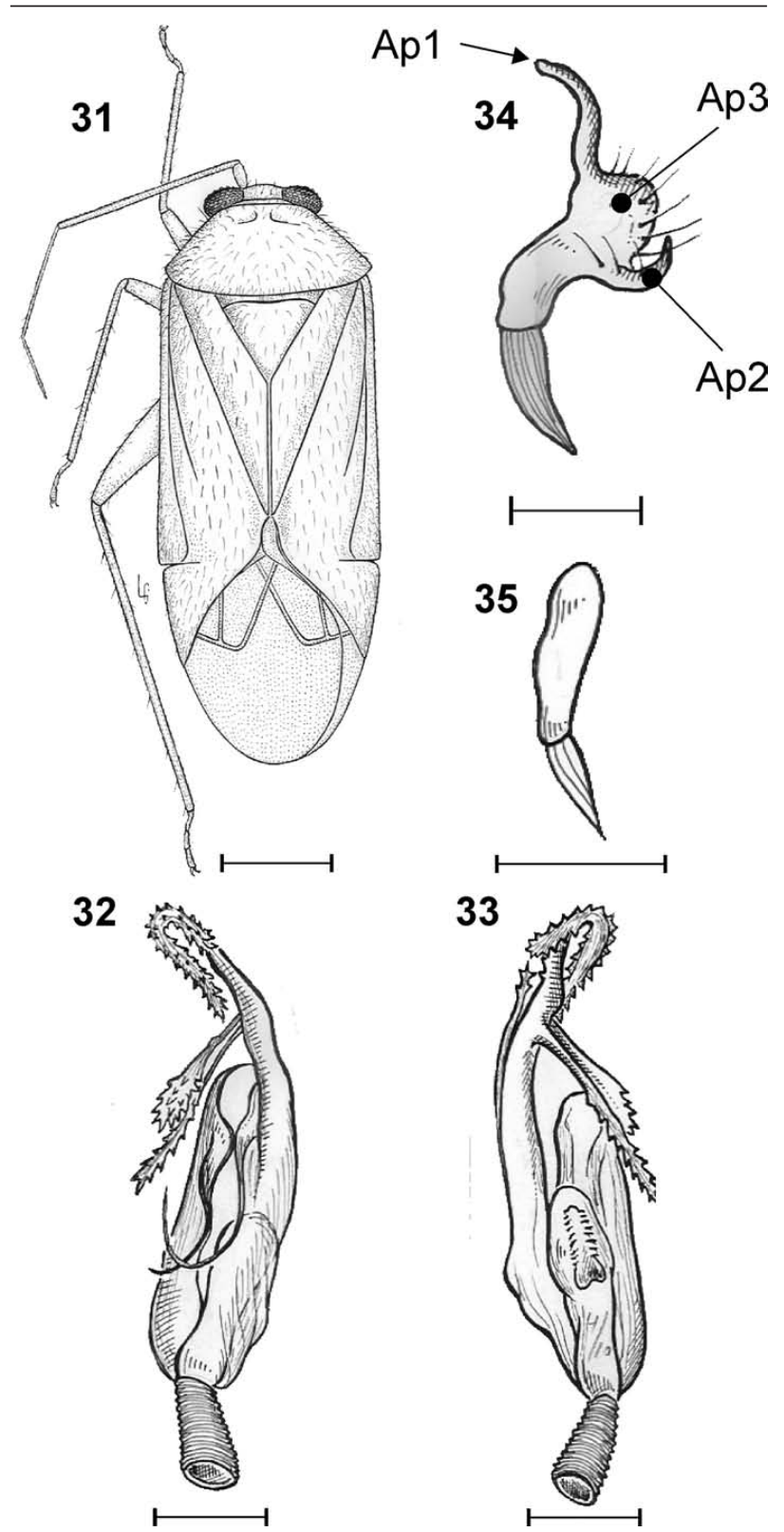

Figures 31-35

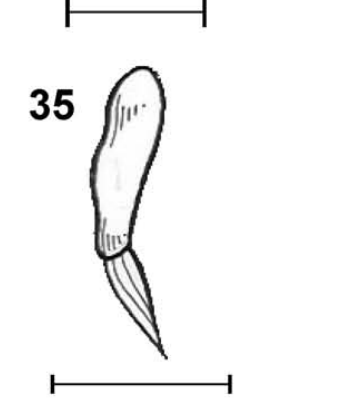

Guianocoris unicolor n. g., n. sp. 31, Habitus en vue dorsale. 32-33, Endophallus en vue latéro-ventrale et latéro-dorsale respectivement. 34, Paramère gauche en vue latéro-dorsale. Ap1 : apophyse primaire, Ap2: apophyse secondaire, Ap3 : apophyse tertiaire. 35, Paramère droit en vue dorsale [Echelles $=0,5 \mathrm{~mm}$ (habitus); 0,1 $\mathrm{mm}$ (génitalias)]. couleur que les deux premiers, leurs soies blanches dressées. Vertex de largeur voisine de celle des yeux, jaune taché de noir, portant un large bourrelet transversal le long de sa marge postérieure, lequel rejoint les yeux. Pas de cou. Rostre basalement jaune, n'atteignant pas la base de l'abdomen. Pronotum. Partie antérieure dépourvue de véritable anneau apical mais portant un bourrelet transversal blanc ivoire au niveau du vertex qui pourrait faire songer à un tel anneau, ce bourrelet lui-même porteur de quelques soies brunes, dressées et se poursuivant sur les rebords latéraux du disque par une très fine marge jaune. Callosités pronotales invisibles (le bourrelet décrit ci-dessus trop antérieur pour en être homologue). Disque pronotal jaune-verdâtre, brillant, ruguleux, très finement ponctué, la ponctuation relativement large mais très superficielle, portant une pilosité blanche sous lumière incidente, souple, semidressée, particulièrement dense sur les bords latéraux. Bord postérieur du pronotum non incurvé médianement, les angles huméraux arrondis. Scutellum plan (affaissé médianement sur l'holotype), jaune-verdâtre unicolore, lisse, sa pilosité similaire à celle du pronotum. Parascutum largement couvert. Pattes allongées, jaune-orangé localement tachées de noir suie, les métafémurs larges, portant une petite pilosité sétiforme blanche semi-dressée, les métatibias portant la même pilosité, des spinules noirs et des épines jaunes claires. Tarses jaune-orangé rembrunis apicalement. Hémélytres jaune-orangé, brillants, allongés, embolium bien marqué, la césure embolio-coriale en $S$ allongé, l'embolium quasi vertical antérieurement. Clavus et endocorie très finement mais très densément ponctués, la ponctuation à la fois extrêmement superficielle et étroite. Pilosité hémélytrale sétiforme blanche semi-dressée. Cunéus plus long que large, mal séparé du paracunéus. Membrane brillante, allongée, jaune brunâtre avec des reflets rosés (sous lumière incidente jaune). Génitalias. Endophallus (figs 32-33) complexe, comprenant d'une part un appendice sclérotisé à 4 branches élargies et denticulées à leurs extrémités apicales respectives, et d'autre part un sclérite prenant naissance à hauteur du gonopore secondaire et se terminant apicalement par deux prolongations lamelliformes pointues. Paramère gauche (fig. 34) semblant intermédiaire entre la structure la plus simple des Orthotylini et la structure classique des Phylini, pourvu d'un lobe sensoriel restreint, d'une apophyse primaire (Ap1) allongée, en pointe émoussée, d'une apophyse secondaire (Ap2) incurvée, allongée, pointue, et d'une apophyse tertiaire (Ap3) bombée. Paramère droit (fig. 35) petit, simple.

Femelle inconnue.

Derivatio nominis. Le nom de la nouvelle espèce réfère à son habitus (vert) unicolore.

Commentaire. Les 8 espèces actuellement décrites dans le genre Cyrtotylus Bergroth 1922 (voir Schuh 1995 : 102) paraissent pouvoir se répartir en 4 «sousgroupes», dont les relations phylogénétiques, ainsi que le rang pour trois d'entre-eux, restent à établir. Cela dépasse largement le cadre du présent travail consacré à la faune de Guyane, d'autant que plusieurs espèces de différents sous-groupes doivent encore être décrites, tant d'Argentine que du Pérou.

Guianocoris unicolor se distingue de Cyrtotylus rubricatus Bergroth 1922 (espèce-type du genre), dont se rapprocherait C. venezuelanus Carvalho 1989 b, par :

(1) la coloration uniforme (les membranes de $C$. 
rubricatus et de C. venezuelanus sont tachées de brun, le scutellum de C. rubricatus porte une tache orange, le pronotum et les hémélytres sont largement tachés de rouge chez $C$. venezuelanus) ;

(2) l'article I des antennes plus court que la largeur du vertex (il est plus long chez $C$. rubricatus et $C$. venezuelanus);

(3) l'appendice sclérotisé phallique à 4 branches phyliformes et denticulées, voisin de ceux que l'on connait chez certains Orthotylus Fieber, 1858 (alors qu'il est spiniforme, double, non denticulé, chez $C$. rubricatus, cf. Carvalho 1946, p. 184, fig. 5).

Guianocoris unicolor se sépare des taxa du sousgroupe de Cyrtotylus catarinensis Carvalho 1989a (C. antoniensis Carvalho 1989a, C. catarinensis Carvalho 1989a et C. ricardoi Carvalho 1988) par :

(1) sa couleur jaune-verdâtre largement uniforme (dépourvue de lignes ou de taches rougeâtres plus ou moins bien définies formant un dessin complexe sur les ailes, le pronotum et la tête) ;

(2) son appendice sclérotisé nettement plus complexe (celui de C. catarinensis est en pointe allongée, l'ouverture du gonopore secondaire étant elle-même beaucoup plus longue et surmontée d'un petit sclérite, cf. Carvalho, op. cit., p. 449, fig. 14), alors qu'il s'en rapproche par la longueur du premier article antennaire et l'uniformité de la pilosité.

Enfin, Guianocoris unicolor s'éloigne très nettement du sous-groupe de C. wygodzinskyi Carvalho 1950 (C. wygodzinskyi Carvalho 1950 et C. cruciatus Carvalho \& Carpintero 1991b), à la fois par :

(1) sa coloration jaune-verdâtre uniforme, membrane comprise (le clavus, mais aussi la corie de C. wygodzinskyi sont porteurs de larges bandes rouges, la membrane $C$. cruciatus étant porteuse de dessins bruns complexes tandis que clavus et corie de cette espèce présentent, sur le vivant, des dessins gris similaires à ceux de $C$. wygodzinskyi qui s'estompent en collection);

(2) sa pilosité simple (alors qu'elle est triple, constituée de soies squamiformes, couchées, noires, de soies sétiformes blanches, semi-dressées et de grosses soies noires dressées sur la tête et la partie antérieure du pronotum chez C. cruciatus);

(3) ses paramères plus largement asymétriques.

Compte tenu de la discussion qui précède, nous considérons le genre Cyrtotylus Bergroth 1922, tel qu'actuellement défini dans la littérature sur base de ses membres, comme inhomogène et fort probablement polyphylétique. Notre nouveau taxon se sépare clairement des espèces actuellement classées, sous le nom Cyrtotylus, dans chacun des 3 sousgroupes mentionnés ci-dessus. En conséquence, nous proposons le nouveau genre Guianocoris pour $G$. unicolor n. sp. Cyrtotylus ruber Carvalho \& Carpintero 1991a, décrit (pp. 769-770) d'Oran, Salta, Argentine, se caractérise par sa coloration rougeâtre uniforme, par sa pilosité dorsale simple, sétiforme, semi-dressée, par l'article I des antennes plus court que la largeur du vertex, et par la structure des génitalias du mâle (un appendice sclérotisé à 3 branches auquel s'ajoutent des prolongations du gonopore secondaire). La présence simultanée de ces états de caractères nous incite à proposer la nouvelle combinaison Guianocoris ruber (Carvalho \& Carpintero 1991a) nov. comb.

\section{Lundioides $\mathbf{n} . \mathrm{g}$.}

Description. Habitus. Corps oblong, lisse, unicolore, revêtu de longues soies érigées ou semi-érigée (fig. 36). Yeux contigus au pronotum, grands, occupant la quasi totalité de la hauteur de la tête. Front nettement incurvé. Vertex caréné. Premier article antennaire élargi sub-basalement, revêtu de courtes soies érigées, leur longueur inférieure à la largeur de l'article (contrairement aux soies érigées des articles III et IV, plus particulièrement du IV, qui sont elles semi-érigées, comme celles de l'article II). Hémélytres allongés, exocorie bien marquée, élargie apicalement, la césure embolio-coriale en $S$ allongé. Métafémurs plus larges et plus longs que les pro- et mésofémurs ; épines tibiales petites; spinules présents. Phallus avec trois appendices sclérotisés.

Espèce-type. Lundioides couturieri n. sp.

Derivatio nominis. Le nom du nouveau genre rappèle sa similarité d'habitus avec Lundiella Carvalho 1951. Genre grammatical: masculin (en vertu de l'Article 30.1.4.4. du Code International de Nomenclature Zoologique, Commission Internationale de Nomenclature Zoologique, 1999).

Commentaire. Lundioides n. g. est assez voisin de Lundiella Carvalho 1951 (Deraeocorinae) par son habitus, en plus oblong. Il se distingue cependant de Lundiella par la structure des ongles, par celle des génitalias mâles (telle que représentée par Carvalho \& Maldonado 1982), par l'absence de ponctuation pronotale et par son prolongement pygophoral.

Au sein des Orthotylini centre- et sud-américains, Lundioides n. g. se distingue par :

(1) la largeur «restreinte» de ses deux derniers articles antennaires, inférieure à celle des deux premiers et non fusiformes ;

(2) par sa surface dorsale quasi lisse, très finement striolée, dépourvue de soies squamiformes ;

(3) par ses yeux accolés au pronotum dont la marge postérieure - quasi droite - est bien séparée du mésoscutum ;

(4) par sa coloration brune et son premier article antennaire subcylindrique ;

(5) par son scutellum plan ;

(6) ainsi que par son vertex qui, quoique marginé, n'est pas déprimé et porte au plus quelques longues soies semi-dressées. 
La présence simultanée de ces états de caractères conduit au couplet 35 de la clé des Orthotylini néotropicaux de Carvalho (1985b: 250-253). Lundioides n. g. ne correspond strictement à aucune des deux alternatives proposées par Carvalho (loc. cit.). En effet son vertex est marginé et porte quelques longues soies semi-dressées (ce qui conduit au couplet 36) mais il n’est pas déprimé (ce qui devrait conduire au couplet 38).

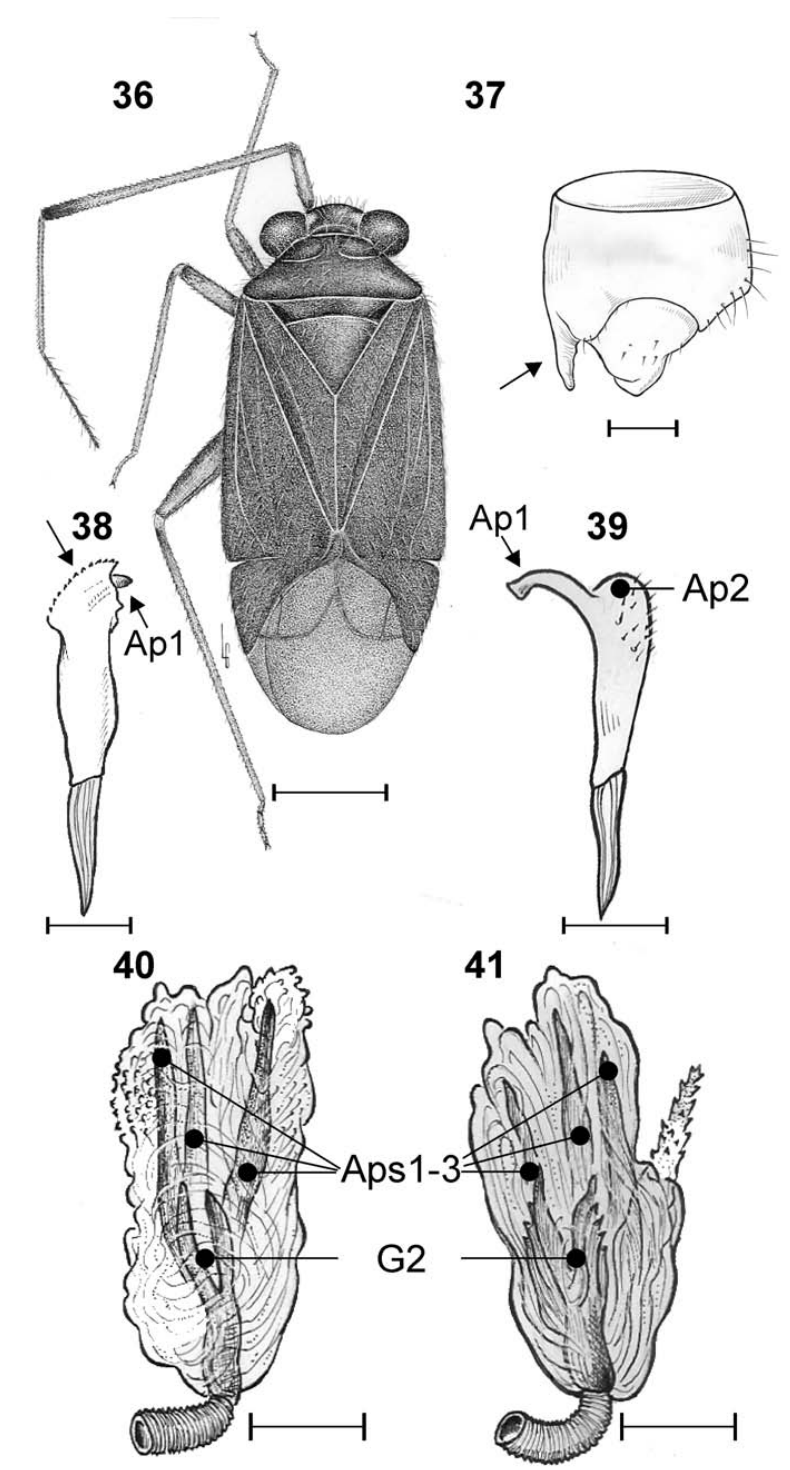

Figures 36-41

Lundioides couturieri n. g., n. sp. 36, Habitus en vue dorsale. 37, Pygophore en vue dorsale. Flèche: prolongation digitiforme allongée. 38, Paramère droit. Flèche : collerette de denticules. 39, Paramère gauche en vue dorsolatérale. Ap1 + Flèches : apophyse primaire, Ap2 : apophyse secondaire. 40-41, Endophallus en vues dorso-latérale [Echelle $=0,5 \mathrm{~mm}$ (habitus); $0,1 \mathrm{~mm}$ (génitalias)].
Des espèces du genre Gaveanus Carvalho 1984b, Lundioides se sépare par son habitus oblong et sa pilosité dorsale plus courte. Des autres genres repris par Carvalho (op. cit. : 252) dans son couplet 38, Lundioides se sépare par la petite taille $(2 \mathrm{~mm})$ et la fracture cunéale profonde.

Des genres repris par Carvalho dans son couplet 36 (op. cit.), Lundioides se rapproche du groupe Hadronemisca Carvalho 1973 - Hadronemella Carvalho 1984b, les hémélytres opaques excluant Pliniella Bergroth 1922. Lundiella n. g. se distingue aisément de Hadronemisca tant par son habitus, en particulier par sa taille inférieure et par sa pilosité dorsale, que par les génitalias mâles (représentés pour $H$. corcovadensis Carvalho \& Gomes 1970a par Carvalho 1977 : 22). De tous les Hadronemella Carvalho 1984b connus, Lundioides $\mathbf{n}$. g. se sépare par la structure des génitalias mâles, notamment par l'apophyse du paramère droit (en pointe simple et non pas évasée, porteuse d'une série de très larges dents) mais aussi par la structure du gonopore secondaire (qui est de "type Orthotylus» classique, en forme de lucarne, chez Hadronemella, contrairement à Lundioides n. g.). Il se sépare en outre par la présence de trois appendices sclérotisés simples (alors que lorsqu'ils sont multiples chez Hadronemella, ils sont généralement de "type Orthotylus», c.-àd. effilés, phyliformes et denticulés; se référer aux figures de Carvalho \& Carpintero 1991a et Carvalho \& Costa 1992) similaires par leur forme aux deux appendices sclérotisés des Falconia Distant 1884 (se référer aux figures de Carvalho 1987). Compte tenu de la discussion ci-dessus, nous proposons Lundioides $\mathbf{n}$. g. pour L. couturieri $\mathbf{n}$. sp., seule espèce actuellement connue. Le classement de ce curieux taxon au sein des Orthotylini sud-américains semble délicat dans l'état actuel de nos connaissances.

\section{Lundioides couturieri $\mathrm{n}$. sp. (Figs 36-41)}

Matériel type : Holotype $\hat{\sigma}$ : «French Guyana : $13 \mathrm{~km}$ SSE of St. Laurent (= Saint Laurent), 8.VI.1986, E. G. Riley \& D. A. Rider» (USNM) [535'ㄷ' W, 5²8’07”N ; fig. 52, point 6].

Description. Mâle (holotype). Mensurations. Longueur totale : 2,1 ; largeur totale : 0,8 . Longueur de la tête en vue dorsale : 0,2 ; largeur du diatone : 0,7 ; largeur du vertex : 0,3 . Longueur des articles antennaires : I : 0,2 ; II : 1,2; III : 0,5; $\mathrm{IV}: 0,3$. Longueur du pronotum : 0,4 ; largeur (postérieure) du pronotum : 0,9 . Longueur du cunéus : 0,6 ; largeur du cunéus : 0,2 . Coloration dorsale marron ; vertex et front médianement brun foncé, avec une bande jaune clair rebordant chaque œil. Tête. Clypéus libre en vue dorsale, vertical, peu nettement séparé du front, rouge-orangé. Joues et brides petites, ni carénées, ni tuberculées, rouge-orangé, les joues ventralement avec une bande jaune. Front court, lisse, quasi vertical et glabre, tout comme le vertex, portant au plus quelques soies sétiformes relativement 
longues, semi-dressées ou dressées. Vertex et front médianement brun foncé, avec une bande jaune clair rebordant chaque œil. Yeux glabres, grands, occupant les $3 / 4$ de la hauteur de la tête en vue frontale et pratiquement toute sa hauteur en vue latérale, rouge-rosé tachés de noir. Antennes jaune clair, l'article II brun à l'apex, légèrement épaissi en massue, l'article III lui aussi rembruni à l'apex, le IV brun. Vertex dépourvu d'impression ou de sillon, très finement marginé, la carène continue. Rostre atteignant les coxae postérieurs, rouge basalement, jaunebrun apicalement. Pas de cou. Pronotum brun jaunâtre à marron, taché de rouge-orangé apicalement. Anneau apical mal délimité du disque (singulièrement sur les côtés) comme c'est généralement le cas chez les Orthotylini, lisse, glabre, contigu aux callosités. Callosités pronotales lisses, quasi glabres, bien visibles, totalement séparées, n'atteignant pas les rebords latéraux du pronotum. Disque du pronotum pratiquement lisse, très finement strié, quasi glabre, portant, en plus d'une rare pilosité similaire à celle de la tête (surtout concentrée sur la partie antérieure des bords latéraux du pronotum), une paire de soies raides, spiniformes, aux angles antérieurs. Bord postérieur du pronotum non incurvé médianement, quasi droit, les angles huméraux arrondis. Scutellum plan, brun jaunâtre à marron, quasi unicolore, pratiquement glabre et lisse. Mésoscutum, largement découvert, porteur de fossettes latérales, de même couleur que le scutellum. Pattes. Fémurs jaunes tachés de rouge basalement, portant de longues soies blanches semi-dressées. Tibias et tarses jaunes pâles, métatibias portant de petites épines jaunes, de longueur légèrement supérieure à la largeur médiane des métatibias, ainsi que de minuscules spinules noirs. Ongles de type Orthotylini. Hémélytres brun jaunâtre à marron, tachés de rouge-rosé sur l'exocorie. Exocorie bien marquée, élargie apicalement, la césure embolio-coriale en $S$ allongé, tachée de rouge, cette tache cependant peu distincte du reste de l'hémélytre. Corie portant quelques longues soies blanches à argentées semi-dressées. Césure cunéale profonde. Membrane brun foncé, nervures brunes. Face ventrale brun foncé, propleures pâles sur leur marge antérieure, procoxae blanchâtres. Pygophore (fig. 37) portant une prolongation digitiforme allongée (flèche). Génitalias. Endophallus (figs 40-41) complexe, comprenant d'une part trois appendices sclérotisés pointus (Aps 1-3) et d'autre part un lobe couvert de larges denticules. Gonopore secondaire (G2) se prolongeant lui-même par deux lobes pointus. Paramère gauche (fig. 39) pourvu d'un lobe sensoriel restreint, d'une apophyse primaire (Ap1) allongée, en pointe émoussée, d'une apophyse secondaire (Ap2) bombée porteuse de nombreuses soies. Paramère droit (fig. 38) allongé, son apophyse primaire (Ap1) en pointe étroite, son corps portant une "collerette" de denticules.

Femelle inconnue.

Derivatio nominis. Le nom de la nouvelle espèce est proposé en hommage au Dr Guy Couturier de l'antenne IRD du Muséum national d'Histoire naturelle, Paris.

Commentaire. L. couturieri n. sp. se reconnaît aisément à ses structures endophalliques très particulières (présence de 3 appendices sclérotisés de type Falconia, forme unique du gonopore secondaire).

\section{Proboscidotylus nigrosquamis (Maldonado 1969) (Figs 42-47)}

Références. Parthenicus nigrosquamis Maldonado 1969: 68 (comme n. sp.) ; Schuh 1995: 179 (catalogue); Proboscidotylus nigrosquamis: Henry $2003:$ 60, 63-65 (redescription, nouvelle combinaison).
Spécimens examinés. Guyane française : $1 \mathrm{O}^{\Uparrow}: 13 \mathrm{~km}$ SSE de Saint Laurent, 8.VI.1986, E. G. Riley \& D. A. Rider leg. (étiqueté par Carvalho comme holotype de Guinisca pallida n. sp., un nom manuscrit) (USNM). Porto-Rico : $2 \hat{0} \hat{o}$ : Carribean N. F., El. Verde Field Station, 8-14.V.1985, UV, 300m., C.U.I.C. 1985 expédition, E. R. Hoebeke, J. K. Libherr \& S. W. Nichols (USNM, identification T. J. Henry).

Remarque. Ce taxon fut originellement décrit dans le genre Parthenicus Reuter, 1876 de Porto Rico par Maldonado (1969 : 66) puis transféré par Henry (2003 : 62) dans le genre Proboscidotylus Henry 1995. Il était connu de Porto Rico et des Etats-Unis (en l'occurrence, de Floride cf. Henry, 2003 : 59) mais n'avait pas encore été signalé de Guyane française, ni même d'Amérique du Sud. Cette première mention pour le continent nous donne l'occasion d'une redescription, afin de faciliter de futures identifications.

Redescription. Mâle. Mensurations (du mâle de Guyane, suivies entre crochets de la fourchette de valeurs donnée par Henry 2003: 63-64 pour ces mêmes structures, d'après 5 mâles ; *: pas de valeur disponible dans le travail de Henry, op. cit.). Longueur totale : 2,4 [2,35-2,55] ; largeur totale : 1,1 $[1,01-1,09]$. Longueur de la tête en vue dorsale : $0,1\left[{ }^{*}\right]$; largeur du diatone : $0,8[0,57-0,59]$; largeur du vertex : $0,2[0,20-$ $0,21]$. Longueur des articles antennaires : I : 0,2 [0,20-0,21] ; II : $1,0[0,94-0,96]$; III : $0,4[0,35-0,38]$; IV : $0,3[0,22-0,29]$. Longueur du pronotum : $0,2[0,27-0,29]$; largeur (postérieure) du pronotum : $0,9[0,82-0,91]$. Longueur du cunéus : $0,3\left[{ }^{*}\right]$; largeur du cunéus : $0,3{ }^{[*}$. Coloration dorsale jaune à jauneverdâtre. Tête. Clypéus partiellement caché en vue dorsale par le bombement frontal (effondré sur le spécimen de Guyane), vertical, nettement séparé du front, jaune unicolore. Joues et brides petites, ni carénées, ni tuberculées, jaunes unicolores. Front jaune ou jaune-orangé, bombé, le bombement plus marqué en vue frontale que dorsale, médianement sillonné, à peine ponctué, portant quelques soies sétiformes semi-dressées. Vertex jaune à jaune orangé, subtriangulaire, non strié, à peine ponctué, immarginé, portant quelques longues soies sétiformes semi-dressées. Yeux jaunes tachés de rouge-brun ou globalement rouge-brun, glabres, grands, occupant la hauteur de la tête tant en vue frontale que latérale (fig. 43). Antennes jaunes claires, l'article I court et épais, jaune-orangé, l'article II subcylindrique, jaune, taché de gris verdâtre basalement, les articles III et IV jaune-orangé, tous les articles porteurs de courtes soies blanches, sétiformes, semi-dressées, l'article I porteur en outre de quelques soies dressées, plus longues. Rostre atteignant pratiquement, voire dépassant légèrement, les coxae postérieurs, jaune-orangé. Pas de cou. Pronotum jaune, dépourvu de véritable anneau apical comme c'est généralement le cas chez les Orthotylini, ses bords latéraux et antérieurs cependant nettement carénés, la carène antérieure pouvant même former des bourrelets. Pas de véritables callosités pronotales. Disque du pronotum ruguleux, non strié, finement ponctué, une ligne, plus ou moins visible selon les individus, de points superficiels mais relativement larges suivant son bord postérieur. Surface du disque portant de longues soies sétiformes dressées ou semi-dressées, blanches (plus denses sur ses marges latérales) ainsi que de courtes soies noires squamiformes rassemblées par groupes de 4 à 10 soies sur une ligne transversale submédiane antérieure à la ligne de larges points. Bord postérieur du pronotum nettement incurvé concave, les angles huméraux arrondis convexes. Scutellum 
plan, jaune ou jaune-orangé quasi unicolore, pratiquement glabre et lisse. Mésoscutum largement découvert, dépourvu de fossettes latérales, de même couleur que le scutellum. Pattes. Fémurs jaunes ou jaune-orangé, portant de longues soies blanches semi-dressées. Tibias et tarses jaune pâle, métatibias portant en plus de petites épines jaunes, de longueur légèrement supérieure à la largeur médiane des métatibias ainsi que de minuscules spinules. Ongles de type Orthotylini. Hémélytres

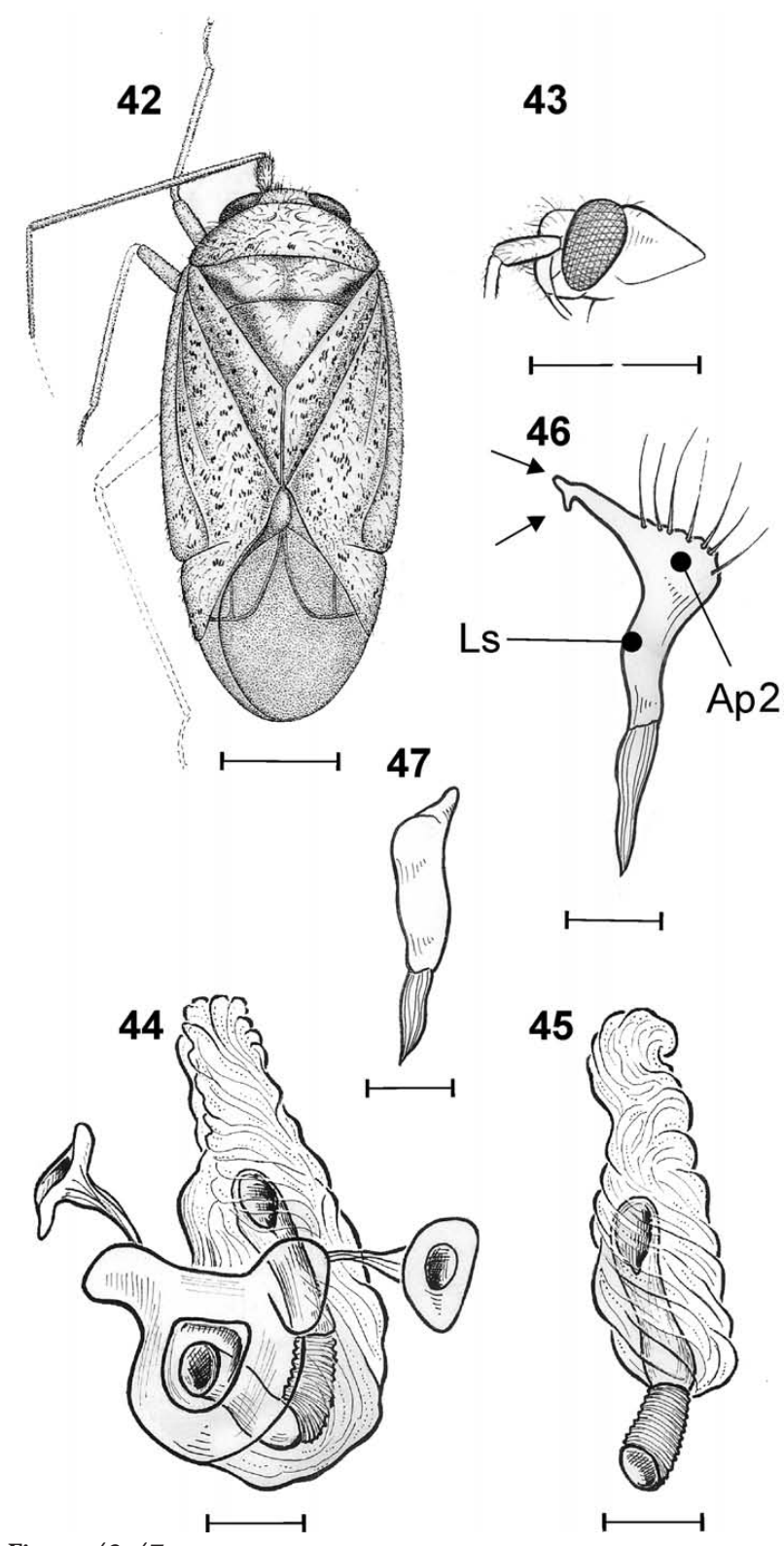

Figures 42-47

Proboscidotylus nigrosquamis (Maldonado 1969). 42, Habitus en vue dorsale. 43, Tête en vue latérale. 44, Phallus en vue dorsale. 45, Endophallus en vue dorso-latérale. 46, Paramère gauche en vue dorsale. Ap2 : apophyse secondaire, Ls : lobe sensoriel, les flèches indiquent les lobes de l'apophyse primaire. 47, Paramère droit en vue dorsale [Echelles $=0,5 \mathrm{~mm}$ (habitus); $0,05 \mathrm{~mm}$ (génitalias)]. ruguleux, finement ponctuées, jaune-verdâtre translucides (particulièrement les endocories), exocories jaune-orangé, opaques sur les $2 / 3$ antérieurs de leur longueur. Clavii et coria portant quelques courtes soies sétiformes blanches à argentées, semi-dressées ou couchées, et de nombreuses soies squamiformes noires, couchées. Cunei largement translucides, jaune-verdâtre, portant une pilosité similaire au reste des hémélytres ainsi qu'un groupe de soies squamiformes noires à la limite des paracunei. Membrane jaunâtre, parfois avec des reflets rosés, nervures jaunes. Face ventrale jaune. Génitalias. Phallus (figs 44-45) très simple, incluant cependant un appendice sclérotisé à peine visible (omis sur la figure). Paramère gauche (fig. 46) pourvu d'un lobe sensoriel (Ls) restreint, d'une apophyse primaire allongée, bifide (flèches), d'une apophyse secondaire (Ap2) bombée porteuse de nombreuses soies. Paramère droit (fig. 47) massif.

Femelle similaire au mâle, le front cependant plus nettement bombé et les hémélytres plus larges selon Henry (2003 : 64).

Commentaire. $P$. nigrosquamis possède clairement les états de caractères diagnostiques du genre Proboscidotylus Henry 1995, tels que synthétisés par Henry (2003 : 59-60), à savoir :

(1) le front fortement bombé ;

(2) le clypeus proéminent (la présence d'un tubercule basal au niveau du tylus étant caractéristique des seuls mâles de $P$. carvalhoi Henry 1995);

(3) l'article I des antennes court et épais ;

(4) l'article II allongé et cylindrique;

(5) la double pilosité pronotale et hémélytrale, incluant des soies sétiformes semi-dressées ou couchées, blanches, mélangées avec des soies squamiformes, noires, couchées (un état de caractère plutôt rare chez les Orthotylini, cf. Henry, op. cit.) ;

(6) une touffe de soies squamiformes noires sur chaque paracunéus ;

(7) des hémélytres largement translucides (ce qui rapprocherait Proboscidotylus du groupe de Zanchius au sens de Schuh 1974);

(8) une série de soies épaisses (probablement glandulaire) sur la surface ventrale du pygophore.

Cette espèce se sépare de $P$. carvalhoi, seule autre espèce connue du genre Proboscidotylus, qui fut décrite du Mexique, par sa longueur totale inférieure, le tylus des mâles nettement moins bombé, l'article II des antennes moins longs (à peine plus long que la largeur du pronotum) et la structure des génitalias.

\section{Phylinae \\ Phylini}

\section{Ranzovius fennahi Carvalho 1954}

Références: Ranzovius fennahi Carvalho 1954a: 93 (comme n. sp.); Henry 1984 : 64 (mention de Guyane française) ; Schuh 1995 : 420 (catalogue).

Remarque : Pas de donnée additionnelle disponible. 


\section{Pilophorini}

\section{Sthenaridea carvalhoi Schuh \& Schwartz 1988 (Figs 48-51)}

Références. Sthenaridea carvalhoi Schuh \& Schwartz 1988 : 187-188, 194 (comme n. sp.) ; Schuh 1995 : 471 (catalogue).

Spécimens examinés. Guyane française : $1 \uparrow$ : Route nationale $\mathrm{n}^{\circ} 2$ vers Régina, $67 \mathrm{~km}$ sud de Cayenne, 06.VI.1986, E. G. Riley \& D. A. Rider. $2 \widehat{\jmath}, 8$, 8 우 : Route nationale $\mathrm{n}^{\circ} 2$ vers Régina, $15 \mathrm{~km}$ sud de Cayenne, 02.VI.1986, E. G. Riley \& D. A. Rider (MNRJ). $4 \delta^{\lambda}$ : Extension de la route nationale $\mathrm{n}^{\circ} 1,20 \mathrm{~km}$ au sud-est de Saint Laurent, 07.VI.1986, E. G. Riley \& D. A. Rider (MNRJ, USNM : un mâle étiquetté comme «holotype» par Carvalho). 1ð̄, 4 우 : Extension de la route nationale $\mathrm{n}^{\circ} 1$ vers Saint Laurent, $13 \mathrm{~km}$ au sud sud-est de Saint Laurent, 08.VI.1986, E. G. Riley \& D. A. Rider. 19 : Montagne des Singes, près de Kourou, 03.VI.1986, E. G. Riley $\&$ D. A. Rider. $1+$ : route nationale $\mathrm{n}^{\circ} 1$ vers Saint Laurent, $9 \mathrm{~km}$ au sud-est d'Iracoubo, 07.VI.1986, E. G. Riley \& D. A. Rider (MNRJ) (tous collectés à la lampe à vapeur de mercure). Nota: Le spécimen désigné comme holotype par Schuh \& Schwartz (1988 : 189) provient, lui, du Pérou, Puerto Bermudez et est préservé dans les collections de la Cornell University, à Ithaca, New York, United States of America.

Remarque. Sthenaridea carvalhoi Schuh \& Schwartz 1988 est une espèce nouvelle pour la faune de Guyane largement répandue en Amérique Centrale (y compris les Caraïbes) et en Amérique du Sud. Elle est en effet connue du Belize, du Brésil (Amazonas, Rio de Janeiro), du Costa Rica, de Cuba, de République Dominicaine, de Guadeloupe, du Guyana, de Jamaïque, du Mexique, du Pérou, du Surinam et de Trinidad et Tobago (Schuh \& Schwartz 1988 : 188). Sa découverte en Guyane ne constitue donc pas une surprise. Il nous parait cependant utile de la redécrire pour faciliter de futures identifications.

Redescription. Mâle. Mensurations (d'un mâle de Guyane de l'USNM étiqueté par Carvalho comme «holotype», suivies entre crochets de la fourchette de valeurs donnée par Schuh \& Schwartz, 1988 : 194, table 1, pour ces mêmes structures, d'après 6 spécimens; ${ }^{*}$ : pas de valeur disponible dans le travail de Schuh \& Schwartz, op. cit.). Longueur totale : 2,0 [Schuh \& Schwartz, op. cit., ne reprennent pas la longueur totale mais la longueur de l'apex du tylus à la fracture cunéale : 1,33-1,71] ; largeur totale : 0,9 [*]. Longueur de la tête en vue dorsale : $0,2\left[^{*}\right]$; largeur du diatone : $0,7[0,51-0,66]$; largeur du vertex : $0,3[0,28-0,36]$. Longueur des articles antennaires : I : $0,16\left[{ }^{*}\right]$; II : $0,6[0,53-0,70]$; III : $0,4\left[{ }^{*}\right]$; IV : $0,2\left[{ }^{*}\right]$. Longueur du pronotum : $\left.0,3{ }^{*}\right]$; largeur (postérieure) du pronotum : $0,8[0,66-0,86]$. Longueur du cunéus : 0,24 $\left.{ }^{*}\right]$; largeur du cunéus : $0,2\left[{ }^{*}\right]$. Coloration dorsale et des appendices jaune dorée à jaune-brunâtre. Tête. Clypéus partiellement caché en vue dorsale par le bombement frontal, vertical, nettement séparé du front, jaune unicolore. Joues et brides ni carénées, ni tuberculées, jaunes unicolores. Front jaune, portant quelques soies sétiformes semi-dressées. Vertex jaune, non strié, à peine ponctué, immarginé, portant quelques longues soies sétiformes semi-dressées. Yeux rouge-brun, glabres, grands, occupant la hauteur de la tête tant en vue frontale que latérale. Antennes jaunes claires, l'article I relativement court et épais, en forme de massue, l'article II subcylindrique, jaune, plus foncé à l'extrême apex, les articles III et IV noirs, tous les articles porteurs de soies dressées, relativement longues. Rostre dépassant légèrement les coxae postérieurs, jaune, plus foncé à l'apex. Pas de cou. Pronotum jaune, dépourvu de véritable anneau apical. Callosités pronotales invisibles. Disque du pronotum lisse, non strié, finement ponctué. Surface du disque portant des soies sétiformes semi-dressées ou couchées, blanches (plus denses sur ses marges latérales) ainsi qu'une paire de soies raides près de ses angles antéro-latéraux. Bord postérieur
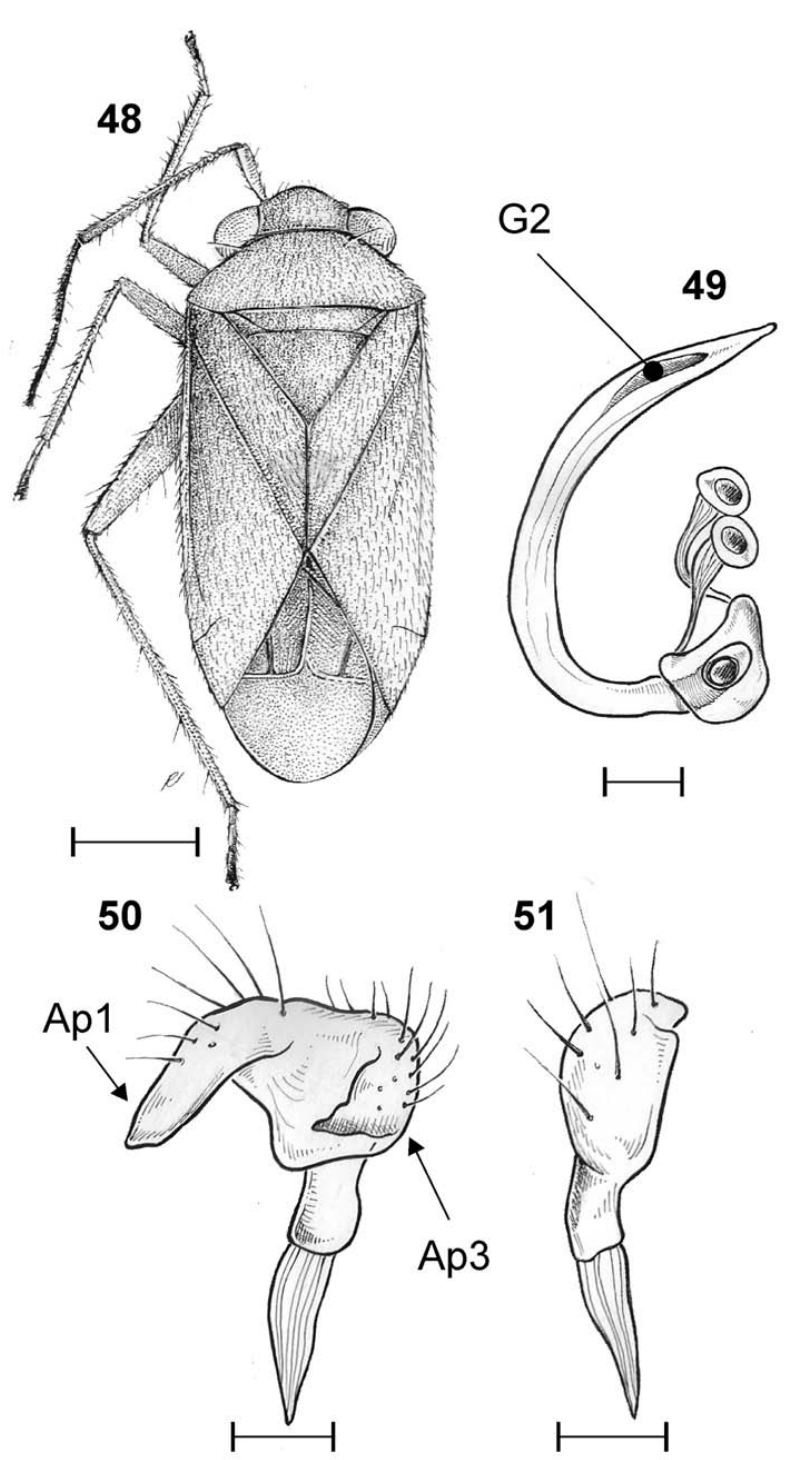

Figures 48-51

Sthenaridea carvalhoi Schuh \& Schwartz 1988. 48, Habitus en vue dorsale. 49. Endophallus en vue latéro-dorsale. G2 : gonopore secondaire. 50, Paramère gauche en vue dorsale. Ap1 : apophyse primaire, Ap3 : apophyse tertiaire. 51, Paramère droit en vue dorsale [échelles $=1 \mathrm{~mm}$ (habitus); $0,05 \mathrm{~mm}$ (génitalias)]. 
du pronotum quasi droit médianement, faiblement oblique latéralement, les angles huméraux anguleux. Pleures portant des soies squamiformes couchées, brillantes, argentées. Scutellum plan, jaune quasi unicolore, portant la même pilosité que le pronotum. Mésoscutum largement découvert, pourvu de fossettes latérales, de même couleur que le scutellum. Pattes. Coxae et fémurs jaunes, portant de longues soies blanches semidressées. Tibias et tarses jaunes, métatibias portant des épines noires, de longueur légèrement supérieure à la largeur médiane des métatibias, ainsi que des spinules noirs. Hémélytres finement ponctuées, jaunes, faiblement brillantes portant de courtes soies sétiformes blanches à argentées semi-dressées ou couchées, plus longues sur l'exocorie. Cunei larges, portant une pilosité similaire au reste des hémélytres. Membrane jaunâtre, nervures jaunes. Face ventrale jaunâtre, sa coloration moins marquée que celle de la face dorsale. Génitalias. Phallus (fig. 49) en forme de $\mathrm{C}$ allongé, son gonopore secondaire en position apicale et bien marqué. Paramère gauche (fig. 50) classique pour un Phylinae, son apophyse primaire (Ap1) large. Paramère droit (fig. 51) massif.

Femelle similaire au mâle.

\section{Discussion}

La faune de Miridae actuellement connue de Guyane française est donc portée à 46 espèces. Il pourrait

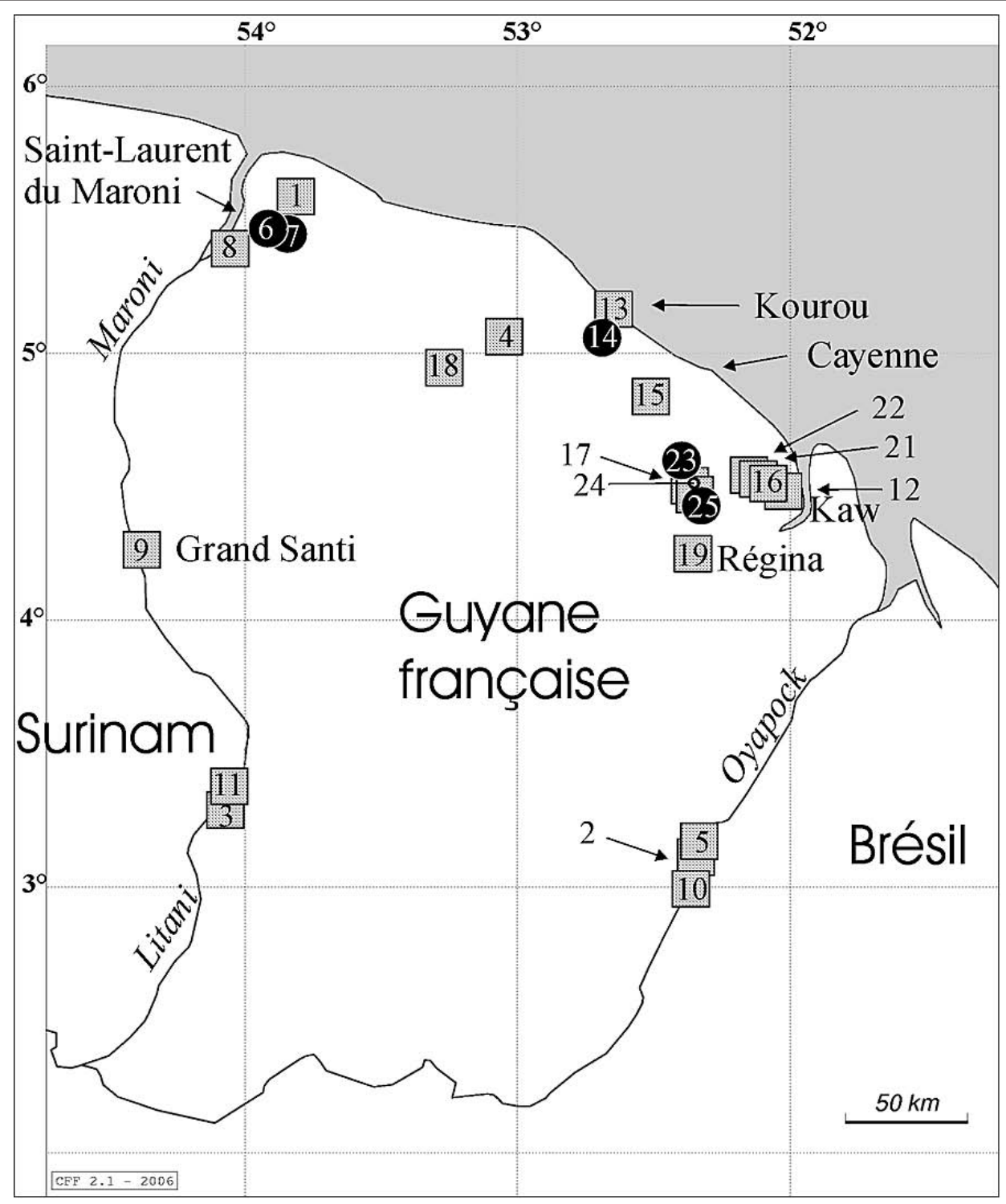

Figure 52

Carte des localités mentionnées dans le présent travail. Les localités-types des nouveaux taxa sont représentées par un rond. Il s’agit des localités 6: Lundioides couturieri n. g., n. sp., 7: Neella guiana n. sp., 14: Phytocoris jugatus n. sp., 23: Phytocoris guianus n. sp., et 25: Diplozona emboliata n. sp. et Guianocoris unicolor n. g., n. sp. Les autres localités sont représentées par un carré. Détail dans le texte et en annexe 2. 
paraître tentant, au terme de ce travail, de tacher de proposer une première analyse biogéographique.

Morone (2001) dans son travail sur la biogéographie de l'Amérique Latine et des Caraïbes, dans lequel il divise cet ensemble en 3 régions, 8 sous régions et 70 provinces biogéographiques, classe la Guyane française dans sa région Néotropicale, sous-région Amazonienne, province de la Guyane humide, sur base tant de la végétation que de la faune. Cela lui permet de rapprocher les Guyanes, le Surinam, une partie du nord du Brésil ainsi que du sud du Venezuela de trois autres de ses provinces biogéographiques, toutes de la sous-région amazonienne: Napo (nord du Pérou, sudouest de la Colombie et est de l'Equateur), Ucayali (Est du Pérou, nord de la Bolivie, et ouest du Brésil) et Para (nord ouest du Brésil).

Une telle analyse biogéographique restreinte à l'échelle de la famille des Miridae, pouvant ou non confirmer des hypothèses antérieures telles celles de Morone (op. cit.) apparaît cependant prématurée aux auteurs, pour les raisons suivantes :

(1) Tant la richesse spécifique que la biogéographie de l'ensemble des Miridae des régions Néotropicale et Andine restent mal connues (Chérot et al. 2007).

(2) Ainsi que mentionné dans l'introduction, la richesse spécifique des Miridae de Guyane française est très certainement largement sous-estimée et le territoire du Département très mal inventorié.

Divers éléments étayent ces affirmations. Les 25 points de collecte des spécimens cités dans le présent travail (fig. 52, annexe 2), qui incluent la totalité de ceux mentionnés par Carvalho \& al. (2001), sont limités : - sur le bord de quelques routes ou chemins principaux (routes nationales 1 et 2 , départementale 5 et départementale 6 jusqu’à Kaw, piste Coralie, piste Crique Serpent et piste de Bélizon totalisent 11 points de collecte) notamment au nord-est et à l'est du Département ; - sur le bord de quelques cours d'eau important, à l'extrême nord-ouest du Département sur le bord du fleuve Maroni et de ses affluents (forêt de Balaté, ile de Twenké) et à son extrême est sur le bord de l'Oyapock (Alicoto, Camopi, ile Massikiri) [se référer à la carte (fig. 52) et à la table 1 de l'annexe 2]. Une grande partie du territoire forestier n'a pas encore été visité et aucune collecte de Cayenne n'est mentionnée.

Parmi les points de collecte cités à la table 1 de l'annexe 2, en un seul endroit (le point 23 sur la fig. 52 , correspondant à la route nationale 2 vers Régina, $45 \mathrm{~km}$ de Cayenne) 9 espèces furent collectées, soit pratiquement $1 / 5$ de la faune miridologique connue de Guyane, tandis qu'en 10 autres endroits une seule espèce l'était. (c) En outre, la prospection en cours de nouvelles localités continue d'apporter un abondant matériel qui inclu des espèces nouvelles pour la Guyane.

On peut néanmoins souligner, en l'état actuel des connaissances, des similarités entre la faune de Guyane française et celle du bassin amazonien (correspondant en partie aux provinces d'Amapa et de la Varzea au sens de Morone, op. cit.), des espèces comme Horcascinus signoreti ou Iridopeplus pellucidipennis en faisant partie. D'autre part, une série d'espèces citées dans le présent travail comme nouvelles pour la faune de Guyane française avaient été décrite du Mato Grosso.

L'état actuelle encore fragmentaire des connaissances de la faune des Miridae de Guyane française devrait conduire les Hémiptéristes d'une part à une analyse plus approfondie des collections déjà disponibles notamment dans les Musées du Nouveau Monde (United States Museum of Natural History, Washington DC, U.S.A., American Museum of Natural History, New York, U.S.A., Museu Nacional, Rio de Janeiro, Brésil...) mais aussi dans les collections privées, encore sous-exploitées, et d'autre part, sur le terrain, à visiter davantage les vastes zones de Guyane largement inexplorées d'un point de vue entomologique (voir fig. 52) et éventuellement à utiliser d'autres techniques de collecte (fogging, piège malaise...).

Remerciements. Les auteurs remercient tous ceux sans l'aide desquels la réalisation de ce travail aurait été impossible. M. le Dr T. Henry (USNM), M. R. Garrouste (MNHN), M. le Dr E. Guilbert (MNHN), M. A. Matocq (MNHN), Mme le Dr D-P. Sigwalt (MNHN) et M. le Dr M. Webb (BMNH) ont bien voulu transmettre au deuxième auteur une partie non négligeable des spécimens objets du présent travail. M. D. Forero (AMNH), M. le Dr I. M. Kerzhner (Zoological Institut, St Petersburg, Russia), M. A. Matocq et Mme le Dr D Pluot-Sigwalt lui ont en outre fourni de précieuses informations (in litt.). Monsieur le Dr Yvan Barbier (Centre de Recherche Nature, Forêt, Bois, Ministère de la Région wallonne, Gembloux, Belgique) lui permis de réaliser la carte des localités-types. Enfin madame le Dr D. Pluot-Sigwalt, M. A. Matocq (MNHN) et M. O.S.G. Pauwels (IRSNB, Smithsonian Institution) ont bien voulu relire notre manuscrit.

\section{Références}

Berg C. 1883. Addenda et emendanda ad Hemiptera Argentinae (2). Anales de la Sociedad Cientifica Argentina 16: 5-32.

Bergroth E. 1910. On some Miridae from French Guiana. Annales de la Société entomologique de Belgique 54: 60-68.

Bergroth E. 1922. New Neotropical Miridae (Hem.). Arkiv för Zoologi 14 (21): 1-14.

Carpintero D.L., Dellapé P. M., Cecila Melo M., 2006. New records of Heteroptera (Hemiptera) from Argentina. Zootaxa 1129: 1-22.

Carvalho J.C.M. 1946. Mirídeos Neotropicais, XXIV : Gêneros Cyrtotylus Bergroth, Peritropis Uhler e Poeas Distant. Boletim do Museu Nacional 67: 171-185.

Carvalho J.C.M. 1950. Mirídeos Neotropicais, XXXVII : Descrição de tres especies novas (Hemiptera). Anais da Academia Brasileira de Ciencias 22: 19-24. 
Carvalho J.C.M. 1951. Neotropical Miridae 44. On a Historical Collection made by P. W. Lund and J. T. Reinhardt in Brazil (Hemiptera). Entomologiske Meddelelser 26: 130-136.

Carvalho J.C.M. 1954a. Neotropical Miridae 67: Genus Ranzovius Distant, predacious on eggs of Theridion. The Annals and Magazine of Natural History 12 (4): 92-96.

Carvalho J.C.M. 1954b. Neotropical Miridae, LXI : Genus Neella Reuter with description of seven new species (Hemiptera). Boletim do Museu Nacional, Zoologia 122: 2-19.

Carvalho J.C.M. 1954c. Neotropical Miridae, LXXIV : Genus Cyrtocapsus Reuter with description of four new species (Hemiptera). Bulletin of the Brooklyn Entomological Society 49: 12-17.

Carvalho J.C.M. 1955. Neotropical Miridae, LXIV : New Bugs of the subfamily Cylapinae. Proceedings of the United States National Museum 103 (3337): 621-632.

Carvalho J.C.M. 1959. Catálogo dos Mirídeos do Mundo. Part IV. Subfamília Mirinae. Arquivos do Museu Nacional XLVIII: 1-384.

Carvalho J.C.M. 1972. Mirídeos Neotropicais, CXLVI : Gênero Monalonion H.-S. (Hemiptera). Anais da Academia Brasileira de Ciencias 44 (1): 119-143.

Carvalho J.C.M. 1973. Neotropical Miridae CXLIX: Hadronemisca gênero novo da Tribo Orthotylini. Revista Brasileira de Biologia 33 (1): 19-20.

Carvalho J.C. M. 1975a. Mirídeos Neotropicais, CXCII: Descrição de dois Subgêneros e Espécies novas do Gênero Notholopus Bergroth (Hemiptera). Revista brasileira de Biologia 35 (3): 369-378.

Carvalho J.C.M. 1975b. Mirídeos Neotropicais, CLXXXIX: Descriçōes de Espécies novas de Poeas e Taedia (Hemiptera). Revista Brasileira de Biologia 35 (2): 167-206.

Carvalho J.C.M. 1975c. Mirídeos Neotropicais, CXCIV: Descrições de dois Gêneros e algumas Espécies novas (Hemiptera). Revista brasileira de Biologia 35 (3): 499-508.

Carvalho J. C.M. 1976a. Mirídeos neotropicais, CCIII: Maxacalinus n. g., com descrição de três especies novas (Hemiptera). Revista Brasileira de Biologia 36 (4): 759-764.

Carvalho J. C. M. 1976b. Mirídeos Neotropicais, CC: Revisão do Gênero Horcias Distant, com Descriçōes de Espécies novas (Hemiptera). Revista Brasileira de Biologia 36 (2): 429-472.

Carvalho J.C.M. 1976c. Mirídeos Neotropicais, CCIV: Descriçôes de quatro espécies novas do Brasil (Hemiptera). Revista Brasileira de Biologia 36 (4): 765-771.

Carvalho J. C. M. 1976d. Mirídeos Neotropicais, CCII: um novo gênero e cinco espécies novas da coleção da Academia de Ciências da Califórnia (Hemiptera). Revista Brasileira de Biologia 36 (3): 715-721.

Carvalho J.C.M. 1977. Mirídeos Neotropicais, CCIX: Descriçōes de quatro espécies novas e do macho de Hadronemisca corcovadensis Carvalho \& Gomes. Revista Brasileira de Biologia 37 (1): 17-22.

Carvalho J.C.M. 1984a. Mirídeos Neotropicais, CCXLII: Descriçōes de oito Espécies novas da Amazonia (Hemiptera). Revista Brasileira de Biologia 44 (1): 99-110.

Carvalho J. C. M. 1984b. Mirídeos Neotropicais, CCXLI: Cinco Gêneros e sete Espécies novos (Hemiptera). Revista Brasileira de Biologia 44 (1): 47-57.

Carvalho J.C.M. 1985a. Mirídeos Neotropicais, CCLXIV: Descriçôes de quatorze Espécies Novas do gêneros Henicocnemis Stål, Neostenotus Reuter e Dagbertus Distant (Hemiptera). Revista Brasileira de Biologia 45 (4): 669-686

Carvalho J. C. M. 1985b. Mirídeos Neotropicais, CCLII: Descriçóes de novos gêneros e espécies da tribo Orthotylini Van Duzee (Hemiptera). Revista Brasileira de Biologia 45 (3): 249-298.

Carvalho J.C.M. 1985c. Mirídeos Neotropicais, CCLXI: Descriçôes de um Gênero e onze Espécies Novos da América Central e América do Sul (Hemiptera). Revista Brasileira de Biologia 45 (4): 653-668.
Carvalho J.C.M. 1986. Mirídeos Neotropicais, CCXXXVI: Um novo Gênero e três Espécies novas da região do Rio Doce (Hemiptera). Revista Brasileira de Biologia 46 (1): 43-48.

Carvalho J.C.M. 1987. Mirídeos Neotropicais, CCLXXXIII: Espécies Sul-Americanas do Gênero Falconia Distant (Hemiptera). Revista Brasileira de Biologia 47 (4): 597-614.

Carvalho J. C.M. 1988. Mirídeos Neotropicais, CCXCIX: Novos Gêneros e Espécies da Região Néotropica. Revista Brasileira de Biologia 48 (4): 873-887.

Carvalho J.C.M. 1989a. Mirídeos Neotropicais, CCCIV: Novos Gênero e Espécies do Brasil. Revista Brasileira de Biologia 49 (2): 443-460.

Carvalho J.C.M. 1989b. Mirídeos Neotropicais, CCCVIII: Gênero e Espécies novos da Venezuela e Colômbia com correçôes taxonomicas. Revista Brasileira de Biologia 49 (2): 461-484.

Carvalho J.C.M., Carpintero D.L. 1991a. Mirídeos Neotropicais, CCCXXXVII: Descripción de doce especies nuevas de Orthotylynae (sic) (Hemiptera). Revista Brasileira de Biologia 51 (4): 763-780.

Carvalho J.C.M. Carpintero D.L. 1991b. Mirídeos Neotropicais, CCCXLVI: Descripcion de un Genero y Siete Especies Nuevas de la Subfamilia Orthotylinae, con notas y correciones taxonomicas (Hemiptera). Anais da Academia Brasileira de Ciencias 63 (2): 33-42.

Carvalho J. C. M., Afonso C. R.S. 1977. Mirídeos Neotropicais, CCVIII: Sobre uma coleçáo enviada para estudio pela Academia de Ciências da California. Revista brasileira de Biologia 37 (1): 7-16.

Carvalho J.C.M., Costa L. L.A. 1990. Mirídeos Neotropicais, CCCXIV: Gênero Diplozona Van Duzee com descrição de espécies novas (Hemiptera). Revista Brasileira de Biologia 52 (1): 175-186.

Carvalho J.C.M., Costa L.L.A. 1992. Mirídeos Neotropicais, CCCXXXVIII: Novos Gênero e especies de Mirinae e novas espécies de Orthotylinae (Hemiptera). Revista Brasileira de Biologia 52 (2): 199-207.

Carvalho J. C. M., Costa L. L.A. 1993. Mirídeos Neotropicais, CCCLIV: Novas Espécies de Taedia Distant do Brasil (Hemiptera). Revista Brasileira de Biologia 53 (2): 217-239.

Carvalho J. C. M., Costa L. L.A. 1994. The genus Fulvius from the Americas (Hemiptera: Miridae). Anales del Instituto de Biologia de la Universidad Nacional Autonoma de México, ser. Zoologia 65 (1): 63-135.

Carvalho J.C.M., Costa L.L.A., Chérot F. 2001. Neotropical Miridae, CCCLXXXIII: Additional species from French Guyana, with a description of the male of Peritropoides annulatus Carvalho 1955 (Hemiptera, Heteroptera, Miridae). Nouvelle Revue d'Entomologie (N. S.) 17 (2): 147-164.

Carvalho J.C.M., Fontes A.V. 1972. Mirídeos Neotropicais, CXLVII: Genero Heniconemis Stål (Hemiptera). Revista Brasileira de Biologia 32 (4): 523-532.

Carvalho J.C.M., Gomes I.P. 1970a. Mirídeos Neotropicais, CXX: descriçóes de um gênero e quatro espécies novos (Hemiptera). Revista Brasileira de Biologia 30 (4): 595-599.

Carvalho J.C.M., Gomes I.P. 1970b. Mirídeos Neotropicais, CXIV: Algumas Espécies de Phytocoris Fallén da Argentina, Brasil, Colombia e Paraguai. Revista Brasileira de Biologia 30 (1): 115-136.

Carvalho J. C. M., Gomes I. P. 1971. Mirídeos Neotropicais, CXXI: Revisão do Gênero Taedia Distant 1883, na Regiáo Neotrópica (Hemiptera). Anais da Academia brasileira de Ciencias 43 (1): 249-286.

Carvalho J. C. M., Jurberg J. 1974. Neotropical Miridae, CLXXX: on the Horcias complex (Hemiptera). Revista Brasileira de Biologia 34 (1): 4965.

Carvalho J.C.M., Jurberg J. 1976. Mirídeos Neotropicais, CCVI: Revisão do Gênero Horciasinus Carvalho \& Jurberg (Hemiptera). Revista Brasileira de Biologia 36 (4): 811-834.

Carvalho J.C.M., Maldonado Capriles J. 1982. Mirídeos Neotropicais, CCXXVIII: Genus Lundiella Carvalho with description of new species (Hemiptera). Revista Brasileira de Biologia 43 (2): 147-150. 
Carvalho J. C. M., Rosas A. F. 1965.Mirídeos Neotropicais, XCV: Gênero e Espécies nova do Suriname, com uma lista de Espécies coligidas em Paramaribo. Revista Brasileira de Biologia 25 (2): 207-210.

Carvalho J.C.M., Schaffner J. C. 1985. Neotropical Miridae, CCLIV: descriptions of new species and one previously described species of Bryocorinae from Mexico, Central America and Venezuela (Hemiptera). Folia Entomologica Mexicana 64: 3-32.

Chérot F. 2002. Eléments de classification générique et de phylogénie des Mirini (Insecta, Heteroptera: Miridae) avec une discussion préliminaire de la relativité des concepts, de limportance de la notion de classe et de linterdépendance des Ecoles en Taxonomie. Volumes 1 et 2. Thèse de doctorat, ULB. Presses Universitaires de Bruxelles, Bruxelles. 535 p.

Chérot F., Carpintero D.L., Maes J.-M. 2007. Sur une collection de Miridae du Nicaragua (Insecta, Heteroptera). Nouvelle Revue d'Entomologie (N.S.) 23 (2): 173-190.

Commission Internationale de Nomenclature Zoologique. 1999. Code International de Nomenclature Zoologique. Quatrième édition adoptée par l'Union internationale des Sciences biologiques. International Trust for Zoological Nomenclature, London. XXIX + 306 p.

Davis N.T. 1955. Morphology of the female organs of reproduction in the Miridae (Hemiptera). Annals of the Entomological Society of America 48: 132-150.

Distant W. L. 1883. Insecta Rhynchota. Hemiptera Heteroptera. vol. I, p. 225264 in Godman F.D., Salvin O (eds). Biologia Centrali Americana. R. H. Porter, London.

Distant W.L. 1883. Insecta Rhynchota. Hemiptera Heteroptera. vol. I, p. 265304 in Godman F.D., Salvin O (eds). Biologia Centrali Americana. R. H. Porter, London.

Distant W.L. 1903. Report on the Rhynchota, Part 1. Heteroptera, p. 223$272+2$ pls in Annandale N., Robinson H. C. Fasciculi malayenses: anthropological and zoological results of an expedition to Perak and the Siamese Malay States, 1901-1902 undertaken by Nelson Annandale \& Herbert C. Robinson under the auspices of the University of Edinburgh and the University of Liverpool. Zoology Part I. Longmans, Green \& Co., London.

Dubois A. 2000. Synonymies and related list in Zoology: general proposals, with examples in herpetology. Dumerilia 4 (2): 33-98.

Fabricius J.C. 1803. Systema Rhyngotorum secundum ordines, genera, species adjectis synonymis, locis, observationibus. Reichard, Brunsvigae. VI + $314 \mathrm{p}$.

Gorczyca J. 2006. The catalogue of the subfamily Cylapinae Kirkaldy 1903 of the World (Hemiptera, Heteroptera, Miridae). Monographs of the Upper Silesian Museum 5: 1-100.

Henry T.J. 1984. Revision of the spider-commensal plant bug genus Ranzovius Distant (Heteroptera: Miridae). Proceedings of the Entomological Society of Washington 86 (1): 53-67.

Henry T.J. 1995. Proboscidotylus carvalhoi, a new genus and species of sexually dimorphic plant bug from Mexico (Heteroptera: Miridae: Orthotylinae). Proceedings of the Entomological Society of Washington 97 (2): 340-45.

Henry T.J. 2003. Proboscidotylus nigrosquamis (Maldonado) (Heteroptera: Miridae: Orthotylinae): new combination and first record for the United States. Proceedings of the Entomological Society of Washington 105 (1): 59-65.
Kelton L.A. 1955. Genera and Subgenera of the Lygus complex (Heteroptera: Miridae). The Canadian Entomologist 87: 277-301.

Maldonado Capriles J. 1969. The Miridae of Puerto Rico (Insecta Hemiptera). Technical paper University of Puerto-Rico 45: 1-133.

Morrone J.J. 2001. Biogeografía de América Latina y el Caribe. Manuales \& Tesis Sociedad Entomológica Aragonesa 3: 1-150.

Poppius B. 1915.- Zur Kenntnis der Indo-Australischen Capsarien. Annales Musei Nationalis Hungarici 13: 1-89.

Reuter O.M. 1876. Capsinae ex America Boreali in Museo Holmiensi asservatae, descriptae. Öfversigt af Kungliga Vetenskapsakademiens Förhandlingar 32 (9) [1875]: 59-92.

Reuter O.M. 1892. Voyage de M. E. Simon au Venezuela (Décembre 1887-Avril 1888). 20 ${ }^{\text {ème }}$ Mémoire. Hémiptères Hétéroptères. 1ère partie. Capsides. Annales de la Société Entomologique de France 61: 391-402.

Reuter O. M. 1902. Miscellanea Hemipterologica. Hemipterologische mitteilungen. Öfversigt af Finska Vetenskaps-Societetens Förhandlingar 44: 141-188.

Reuter O.M. 1908. Eccritotarsus genetivus Distant, eine Cyllocorarie. Wiener Entomologische Zeitung 27: 53-55.

Reuter O.M. 1910. Diagnoses praecursoriae Miridarum divisonis Restheniaria. Annales Historico Naturales Musei Nationalis Hungarici 8: $15-33$.

Sadowska-Woda I., Chérot F., Gorczyca J. 2006. Contribution to the study of the female genitalia of twelve Fulvius species (Heteroptera, Miridae, Cylapinae), p. 617-636 in Rabitsch W. (ed.).- Hug the bug - For the love of true bugs. Festschrift zum 70. Geburtstag von Ernst Heiss. Denisia 19 [Zugleich Kataloge der Oberösterreichischen Landesmuseen, Neue Serie 50].

Schuh R.T. 1974. The Orthotylinae and Phylinae (Hemiptera: Miridae) of South Africa with a phylogenetic analysis of the ant-mimetic tribes of the two subfamilies for the world. Entomologica Americana 47:1-332.

Schuh R.T. 1995. Plant bugs of the world (Insecta: Heteroptera: Miridae). Systematic Catalog, Distributions, Host List, and Bibliography. Entomological Society of New York. XII + 1329 p.

Schuh R. T., Schwartz M. D. 1988. Revision of the New World Pilophorini (Heteroptera: Miridae: Phylinae). Bulletin of the american Museum of Natural History 187 (2): 101-201.

Signoret V. 1858. Note sur les Hémiptères Hétéroptères de la famille des unicellules. Annales de la Société Entomologique de France 3 (6): 499502

Slater J.A. 1950. An investigation of the female genitalia as taxonomic characters in the Miridae (Hemiptera). Iowa State College Journal of Science 25: 1-81.

Stål C. 1859. Hemiptera. Species novas descripsit. Kongliga Svenska Fregattens Eugenies Resa omkring jorden 3: 219-298.

Stål C. 1860. Bidrag till Rio Janeiro-traktens Hemipter-fauna. Kungliga Vetenskapakademiens Handlingar, 2 (7): 1-84 [1858].

Stål C. 1862. Hemiptera Mexicana enumeravit speciesque novas descriptis. Stettiner Entomologische Zeitung 23: 81-118, 289-325.

Stonedahl G. M. 1988. Revision of the Phytocoris Fallén (Heteroptera, Miridae) for Western North America. Bulletin of the American Museum of Natural History 188 (1): 1-257.

Stonedahl G. M., Henry T.J. 1991. A new genus of Mirine plant bugs, Gracilamiris, with three new species from North America. Journal of the New York Entomological Society 99 (2): 224-234. 


\section{Annexe 1. Liste alphabétique provisoire des Miridae de Guyane française}

Cyrtocapsus femoralis Reuter 1892

Dagbertus insignis Carvalho 1977

Diplozona emboliata $\mathbf{n}$. sp.

Eustictus spp.

Fulvius amapensis Carvalho \& Costa 1994

Fulvius bisbistillatus (Stål 1860)

Fulvius breddini Reuter 1902

Fulvius satipoensis Carvalho \& Costa1994

Guianocoris unicolor n. g., n. sp.

Henicocnemis luctuosa (Stål 1860)

Horciasinus signoreti (Stål 1859)

Horciasoides binigrus Carvalho, Costa \& Chérot 2001

Horciasoides gallicus Carvalho, Costa \& Chérot 2001

Horciasoides nigridorsum Carvalho, Costa \& Chérot 2001

Horciasoides punctatus (Carvalho 1976b)

Iridopeplus pellucidipennis Bergroth 1910

Lundioides couturieri $\mathbf{n}$. g., n. $\mathbf{s p .}$

Maxacalinus annulatus Carvalho, Costa \& Chérot 2001

Maxacalinus cuiabanus Carvalho 1976a

Maxacalinus hexapunctatus Carvalho, Costa \& Chérot

2001

Monalonion annulipes Signoret 1858

Neella guiana $\mathbf{n}$. sp.

Notholopus coreoides Carvalho 1975a

Notholopus cuiabanus Carvalho 1975a

Notholopus filicornis (Fabricius 1803)

Peritopoides annulatus Carvalho 1955

Peritropoides guianus Carvalho, Costa \& Chérot 2001

Phytocoris guianus n. sp.
Phytocoris jugatus n. sp.

Poeas castanea Carvalho, Costa \& Chérot 2001

Prepops poppii (Bergroth 1910)

Prepops rollei (Reuter 1910)

Proboscidotylus nigrosquamis (Maldonado 1969)

Ranzovius fennahi Carvalho 1954

Sthenaridea carvalhoi Schuh \& Schwartz 1988

Sysinas fulvicollis (Fabricius 1803)

Taedia bananalensis Carvalho \& Costa 1993

Taedia bilutea Carvalho, Costa \& Chérot 2001

Taedia cajabiana Carvalho 1976c

Taedia coimbrai Carvalho, 1975c

Taedia compactoides Carvalho 1975b

Taedia pauwelsi Carvalho, Costa \& Chérot 2001

Taedia semilota (Stål 1860)

Taedia signata Carvalho \& Gomes 1971

Taedia xinguana Carvalho 1975b 


\section{Annexe 2. Liste alphabetique des lieux de collecte mentionnés dans l'article, avec leurs coordonnées géographiques et le code les symbolisant sur la carte (fig. 52).}

Les localités sont classées par ordre alphabétique et rassemblées lorsqu'elles sont trop voisines pour être représentées séparément sur la carte. Les coordonnées géographiques sont déterminées sur base des informations disponibles sur les étiquettes des spécimens analysés (parfois peu précises) à l’aide du Géoportail de l'Institut Géographique National. * PK : point kilométrique. ** Ce point de collecte n’a pas été identifié. Peut-être s'agit-il d'une route vers la rivière Comté ?

\begin{tabular}{|c|c|c|c|}
\hline Point de collecte & Longitude (W) & Latitude (N) & Numéro \\
\hline Acarouany, auberge du Bois Diable & $53^{\circ} 48^{\prime} 44^{\prime \prime}$ & $5^{\circ} 35^{\prime} 18^{\prime \prime}$ & 1 \\
\hline Alicoyo (= Alicoto), Oyapock & $52^{\circ} 20^{\prime} 44^{\prime \prime}$ & $3^{\circ} 06^{\prime} 40^{\prime \prime}$ & 2 \\
\hline Antécumépata (= Antécume Pata), Saut Kialo & $54^{\circ} 04^{\prime} 14^{\prime \prime}$ & $3^{\circ} 17^{\prime} 18^{\prime \prime}$ & 3 \\
\hline Barrage de Petit Saut & $53^{\circ} 02^{\prime} 55^{\prime \prime}$ & $5^{\circ} 03^{\prime} 50^{\prime \prime}$ & 4 \\
\hline Camopi, (bord de l') Oyapock & $52^{\circ} 20^{\prime} 00^{\prime \prime}$ & $3^{\circ} 10^{\prime} 17^{\prime \prime}$ & 5 \\
\hline extension route nationale $1,13 \mathrm{~km}$ de St. Laurent & $53^{\circ} 54^{\prime} 52^{\prime \prime}$ & $5^{\circ} 28^{\prime} 07^{\prime \prime}$ & 6 \\
\hline extension route nationale $1,20 \mathrm{~km}$ de St. Laurent & $53^{\circ} 50^{\prime} 32^{\prime \prime}$ & $5^{\circ} 26^{\prime} 53^{\prime \prime}$ & 7 \\
\hline Forêt de Balaté, St-Jean du Maroni & $54^{\circ} 03^{\prime} 12^{\prime \prime}$ & $5^{\circ} 23^{\prime} 35^{\prime \prime}$ & 8 \\
\hline Grand Santi & $54^{\circ} 22^{\prime} 35^{\prime \prime}$ & $4^{\circ} 15^{\prime} 55^{\prime \prime}$ & 9 \\
\hline Ile Massikiri, Oyapock & $52^{\circ} 21^{\prime} 54^{\prime \prime}$ & $2^{\circ} 59^{\prime} 37^{\prime \prime}$ & 10 \\
\hline Itani, île de Touenké (= Twenké) & $54^{\circ} 03^{\prime} 25^{\prime \prime}$ & $3^{\circ} 22^{\prime} 42^{\prime \prime}$ & 11 \\
\hline Kaw & $52^{\circ} 01^{\prime} 36^{\prime \prime}$ & $4^{\circ} 29^{\prime} 03^{\prime \prime}$ & 12 \\
\hline Kourou & $52^{\circ} 38^{\prime} 50^{\prime \prime}$ & $5^{\circ} 10^{\prime} 01^{\prime \prime}$ & 13 \\
\hline Montagne des Singes & $52^{\circ} 41^{\prime} 26^{\prime \prime}$ & $5^{\circ} 03^{\prime} 33^{\prime \prime}$ & 14 \\
\hline Patawa (route départementale 5) & $52^{\circ} 30^{\prime} 40^{\prime \prime}$ & $4^{\circ} 50^{\prime} 21^{\prime \prime}$ & 15 \\
\hline Patawa (route départementale 6) $\mathrm{PK}^{*} 37$ et 37,5 & $52^{\circ} 09^{\prime} 09^{\prime \prime}$ & $4^{\circ} 32^{\prime} 40^{\prime \prime}$ & 16 \\
\hline Piste Coralie, PK 2,2 & $52^{\circ} 22^{\prime} 08^{\prime \prime}$ & $4^{\circ} 30^{\prime} 22^{\prime \prime}$ & 17 \\
\hline Piste Crique Serpent, PK 19,1 & $53^{\circ} 16^{\prime} 07^{\prime \prime}$ & $4^{\circ} 56^{\prime} 49^{\prime \prime}$ & 18 \\
\hline Régina, piste de Bélizon, PK 15 & $52^{\circ} 22^{\prime} 59^{\prime \prime}$ & $4^{\circ} 17^{\prime} 03^{\prime \prime}$ & 19 \\
\hline Route de la Conté (= ? Comté) ${ }^{* *}$ & $?$ & $?$ & Non figuré \\
\hline Route départementale 6 vers Kaw, 33,5-34 km de Roura & $52^{\circ} 07^{\prime} 04^{\prime \prime}$ & $4^{\circ} 31^{\prime} 50^{\prime \prime}$ & 21 \\
\hline Route départementale 6 vers Kaw, 37,5 km de Roura & $52^{\circ} 04^{\prime} 56^{\prime \prime}$ & $4^{\circ} 30^{\prime} 47^{\prime \prime}$ & 22 \\
\hline Route nationale 2 vers Régina, 45 km de Cayenne & $52^{\circ} 23^{\prime} 59^{\prime \prime}$ & $4^{\circ} 36^{\prime} 03^{\prime \prime}$ & 23 \\
\hline Route nationale 2 vers Régina, $62 \mathrm{~km}$ de Cayenne & $52^{\circ} 21^{\prime} 00^{\prime \prime}$ & $4^{\circ} 28^{\prime} 20^{\prime \prime}$ & 24 \\
\hline Route nationale 2 vers Regina, $67 \mathrm{~km}$ de Cayenne & $52^{\circ} 19^{\prime} 37^{\prime \prime}$ & $4^{\circ} 25^{\prime} 48^{\prime \prime}$ & 25 \\
\hline
\end{tabular}

